\title{
Glomerular information processing in Xenopus laevis
}

\author{
Dissertation \\ for the award of the degree \\ 'Doctor rerum naturalium' (Dr. rer. nat) \\ Division of Mathematics and Natural Sciences \\ of the Georg-August-Universität Göttingen
}

within the doctoral program

Molecular Physiology of the Brain (CMPB)

of the Georg-August University School of Science (GAUSS)

Submitted by

Daniëlle de Jong

from Dordrecht, the Netherlands

Göttingen, 2016 


\title{
Thesis Committee
}

\author{
Prof. Dr. Dr. Detlev Schild
}

Institute of Neurophysiology and Cellular Biophysics

\section{Prof. Dr. Walter Stühmer}

Dept. of Molecular Biology of Neuronal Signals

Max Planck Institute for Experimental Medicine

\section{Prof. Dr. Michael Müller}

Dept. Neurophysiology and Sensory Physiology

University Medical Center Göttingen

\section{Members of the examination board}

\author{
Prof. Dr. Martin Göpfert \\ Dept. of Cellular Neurobiology \\ Schwann-Schleiden Research Centre
}

Prof. Dr. Ernst Wimmer

Dept. of Developmental Biology

Johann-Friedrich-Blumenbach-Institute of Zoology and Anthropology

\section{Prof. Dr. André Fiala}

Dept. of Molecular Neurobiology of Behaviour

Schwann-Schleiden Research Centre

Date of the oral examination: $\mathbf{2 2}^{\text {nd }}$ of April, 2016 
I hereby declare that the $\mathrm{PhD}$ thesis entitled

"Gomerular information processing in Xenopus laevis""

was written independently and with no other sources and aids than quoted.

Daniëlle de Jong

Göttingen, 22 ${ }^{\text {nd }}$ February 2016 


\section{Contents}

1 Introduction 1

1.1 Glomerular morphology and function $\ldots \ldots \ldots$. . . . . . . . 1

1.2 Shaping odorant responses . . . . . . . . . . . . . . 3

1.3 ORN firing dynamics $\ldots \ldots \ldots \ldots$

1.4 Goal of the Thesis . . . . . . . . . . . . . . . . . 8

2 Methods 9

2.1 Sample preparation ...................... 9

2.1.1 Electroporation . . . . . . . . . . . . . . 9

2.1.2 Slicing and whole mount preparation . . . . . . . . . 10

2.2 Solutions and stimuli $\ldots \ldots \ldots 11$

2.3 Imaging of target glomeruli . . . . . . . . . . . . . . . 12

2.4 Experimental conditions . . . . . . . . . . . . . . . 13

2.4.1 Stimulus-induced responses . . . . . . . . . . . . . 13

2.4.2 Spontaneous activity . . . . . . . . . . . 16

2.5 Image processing $\ldots \ldots \ldots \ldots \ldots \ldots$

2.6 Selection of regions of interest . . . . . . . . . . . . . . 18

2.6.1 Manual ROI selection . . . . . . . . . . . . . . 18

2.6.2 Semi-automatic ROI selection . . . . . . . . . . . . 18

2.6.3 Automatic ROI selection . . . . . . . . . . . . . . . . . . 19 


\section{Contents}

2.7 Response analysis . . . . . . . . . . . . . . . . . . 22

3 Results $\quad 23$

3.1 Dose-response profiles . . . . . . . . . . . . . . . 23

3.2 Fluctuations of stimulus-induced responses . . . . . . . . . 30

3.3 Different types of spontaneous activity . . . . . . . . . . . 36

3.4 Presynaptic glomerular morphology and function . . . . . . . . . . 37

4 Discussion $\quad 44$

4.1 Glomerular sensitivity . . . . . . . . . . . . . . . . . . 44

4.2 Odorant responses and spontaneous activity . . . . . . . . . . . 47

4.3 Methods of ROI selection . . . . . . . . . . . . . . . . . . . 48

5 Conclusions and future directions $\quad \mathbf{5 0}$

6 Summary $\quad 51$

$\begin{array}{lll}7 & \text { References } & 53\end{array}$

8 Acknowledgements

9 Curriculum Vitae $\quad 63$ 


\section{List of Figures}

1.1 Amino acid-sensitive cells of Xenopus laevis tadpoles . . . . . . . . . 6

2.1 Sample preparation . . . . . . . . . . . . . . 11

2.2 Experimental design of repetitive odor stimulation . . . . . . . . . . 13

2.3 Experimental design of single amino acid stimulation . . . . . . . . . 14

2.4 Experimental design of dose-response experiments $\ldots \ldots 15$

2.5 Movement correction . . . . . . . . . . . . . . . 16

2.6 Bleach and line correction $\ldots \ldots \ldots \ldots$

2.7 Semi-automatic ROI selection _ . . . . . . . . . . . . . . . . 19

2.8 Spatiotemporalfilter . . . . . . . . . . . . . . . . 19

2.9 Automatic ROI detection . . . . . . . . . . . . . . . . 21

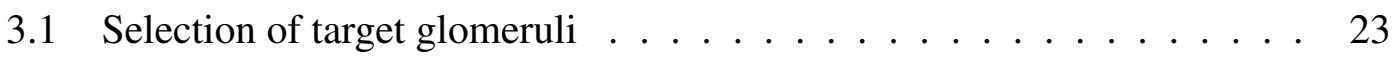

3.2 Differences between intraglomerular dose-response profiles and spontaneous activity patterns . . . . . . . . . . . . 25

3.3 Neighborhood correlation of spontaneous activity . . . . . . . . . 26

3.4 Differences and similarities over subsequent trials . . . . . . . . . 28

3.5 Repetitive stimulation reveals highly similar response profiles . . . . . . 31

3.6 Two example glomeruli with different odorant response profiles . . . . 32

3.7 Intraglomerular odorant response profiles are very similar . . . . . . . 33

3.8 Strong fluctuations during repetitive stimulation with single amino acids 35 
3.9 Spontaneous $\mathrm{Ca}^{2+}$ fluctuations . . . . . . . . . . . . 36

3.10 Signal-to-noise differences between different dyes . . . . . . . . . . . 37

3.11 Visualization of individual intraglomerular axons . . . . . . . . . 39

3.12 Comparison between two ROI selection procedures . . . . . . . . 41

3.13 Automatic detected sequences from experimental data . . . . . . . . . . 42

\section{List of Tables}

3.1 Glomerular response thresholds after stimulation with $10 \mu \mathrm{M}$ AA-mix . 24

3.2 Glomerular response thresholds after stimulation with $100 \mu \mathrm{M}$ AA-mix 24 


\section{Introduction}

Receptor neurons encode and filter signals from the environment for processing by the nervous system. Each sensory system has its own characteristic class of neurons. The receptor neurons of the olfactory system are simply called olfactory receptor neurons (ORNs). They transform an odor into an electrical signal and thus determine the initial encoding of this odor. The resulting odor code is highly complex, as it is affected by many factors, including experience (Reisert and Zhao, 2011), satiety (Aimé et al., 2007, Savigner et al., 2009, Breunig et al., 2010) and age (Rawal et al., 2014). Odorantinduced action potentials are transported via axons of numerous ORNs towards the presynaptic side of neuropils that are located in the olfactory bulb and are also called glomeruli.

\subsection{Glomerular morphology and function}

Olfactory glomeruli are the functional and anatomical units of odor representation in the olfactory bulb (Bozza et al., 2002, Wachowiak et al., 2004). Despite prominent differences in the number of glomeruli, glomerular features seem to be highly conserved across species (Hildebrand and Shepherd, 1997, Imai et al., 2010). One preserved feature I would like to stress here is the consistency in glomerular response maps.

The organization of glomerular response maps indicate that ORN axons do not connect randomly to glomeruli (Drosophila: Couto et al., 2005; mice: Wachowiak and 


\section{Introduction}

Cohen, 2001). As a matter of fact, many factors have been pointed out to be crucial in guiding ORN axons to their target glomerulus (for recent reviews see Takeuchi and Sakano, 2014, Valle-Leija, 2015). The glomerular position, for example, is determined by both external molecular gradients and ORN internal features, such as intracellular cAMP levels (Imai et al., 2006) or the expressed odorant receptor (Feinstein et al., 2004, Mombaerts, 2006). Also in non-mammalian species ORN intrinsic proteins are differentially distributed over the glomerular layer (Manzini et al., 2007b, Braubach et al., 2012). Specifically, Manzini et al. showed that the heterogeneous distribution of presynaptic vesicle proteins is related to distinct glomerular response profiles. Furthermore, in zebrafish a combination of protein and other neuronal markers can even be used to identify single glomeruli (Braubach et al., 2012).

Since ORNs that project to one glomerulus are thought to have the same receptors, single glomeruli might also be identified by their response profiles. The large number of glomeruli complicates the acquisition of a glomerular specific response map in most species. However, over the last decades progress has been made. In 2009, Soucy et al. showed a highly preserved glomerular odor response map in rats and mice.

As described above, single glomeruli and/or glomerular clusters can be distinguished based on their activity patterns, their morphological features or both. This indicates that the features of ORNs projecting to one glomerulus must be fairly similar, although this might reflect maturation (Zou et al., 2004, Manzini and Schild, 2004). On the other hand, the mere fact that numerous ORN axons form the presynaptic side of one glomerulus (Hildebrand and Shepherd, 1997, Byrd and Burd, 1991), indicates intraglomerular differences as well. Some features of ORNs, mainly the ability to change their sensitivities (e.g. Cadiou et al., 2014, Wilson, 2013), inevitably lead to dissimilarities in stimulus-induced responses within single glomeruli. 


\section{Introduction}

In order to estimate the range of stimulus-induced variations within and between intraglomerular ORN populations, it is important to understand how odorant responses are generated, which is described in the following section.

\subsection{Shaping odorant responses}

The first step in odor transduction is the binding of an odor to its receptor, which leads to a conformational change or activation of this protein. Odorant receptors, first described by Buck and Axel (1991) are G-protein-coupled receptors with a broad receptive range. The binding of an odor to its receptor can trigger a cascade of ciliar proteins. There are many reviews and book chapters which describe ORN $2^{\text {nd }}$ messenger pathways in detail (e.g. Schild and Restrepo, 1998, Elsaesser and Paysan, 2007, Ma and Menini, 2010, Pifferi et al., 2009). In general a distinction is made between two classical $2^{\text {nd }}$ messenger pathways, the cAMP (cyclic adenosine mono phosphate) and PLC (phospholipase C) pathway. Those pathways both require a G-protein-coupled receptor. In the cAMP-mediated second messenger pathway, receptor activation results in activation of adenylyl cyclase and consequently triggers cAMP synthesis. After cAMP binding (via calmodulin) cyclic nucleotide gated channels open, allowing $\mathrm{Ca}^{2+}$ and $\mathrm{Na}^{+}$to enter the cell, which results in a depolarization of the membrane potential. By contrast, the PLC-mediated pathway starts with the activation of phospholipase C. PLC cleaves phospoinositide (PIP2), which results in two products, diacylglycerol (DAG) and inositol-trisphosphate (IP3), each serving different functions. The cleaving of PIP2 results in the (in)direct activation of transient receptor potential channels (TRPCs), which is followed by an influx of $\mathrm{Ca}^{2+}$. Amino acid-sensitive ORNs located in the main olfactory epithelium of Xenopus laevis have a PLC-mediated pathway as well (Sansone et al., 2014). 


\section{Introduction}

The transduction machinery of ORNs is rather complex. First of all, various studies indicate that ORNs can have more than one $2^{\text {nd }}$ messenger cascade. Secondly, those pathways can be independently activated (Yu et al., 2014). Thirdly, $2^{\text {nd }}$ messenger compounds can influence cell protein sensitivity, allowing ORNs to modify subsequent responses to stimuli.

Measuring dose response profiles of ORNs or studying responses elicited by repetitive application of the same odor stimulus are common approaches to study stimulusinduced changes of ORN sensitivity. It has been repeatedly shown that $\mathrm{Ca}^{2+}$ is critically involved in adaptation processes occurring in ORNs (Antunes et al., 2014, Spehr et al., 2009, Kurahashi and Menini, 1997). In the classical cAMP-mediated pathway, $\mathrm{Ca}^{2+}$, with the help of calmodulin, affects the sensitivity of cyclic nucleotide-gated channels. Altered ion channel sensitivity mainly influences the receptor potential and the consequent termination of ORN responses (Stephan et al., 2012, Song et al., 2008). Although studies describing adaptation processes usually refer to the cAMP-mediated pathway, they clearly indicate the importance of $\mathrm{Ca}^{2+}$.

The activation of the PLC $2^{\text {nd }}$ messenger pathway also leads to a stimulus-induced $\mathrm{Ca}^{2+}$ influx. It can therefore be expected that $\mathrm{Ca}^{2+}$ is similarly involved in adaptation processes of ORNs with the other canonical pathway, probably influencing ORN firing dynamics as well.

\subsection{ORN firing dynamics}

The fundamental component of neuronal firing is action potentials. Altered ion channel functioning can critically change their shape and occurrence (Bao et al., 2015, Stephan et al., 2012). Bao et al. observed an essential role for $\left[\mathrm{Ca}^{2+}\right]$-activated large-conductance potassium (BK) channels in shaping action potentials of ORNs responding to amino acids. Patch clamp results showed that blocking BK channels results in prolonged re- 


\section{Introduction}

polarization of action potentials and an increased interspike interval at the level of the olfactory epithelium. Furthermore, calcium imaging revealed that amino acid-induced responses were reduced in both ORN cell somata as well as in the axon terminals of ORNs, after blocking BK channels with iberiotoxin.

ORN axon terminals project to the dendrites of mitral cells, forming the pre- and postsynaptic side of glomeruli (Nezlin et al., 2003). Although no amplitude reduction was found at the level of mitral cells, iberiotoxin treatment resulted in a change in response latency (Bao et al., 2015 (calcium imaging)). Since odors are primarily latency-coded in mitral cells (Junek et al., 2010), the results described above show that BK channels are involved in odor coding occurring in amino-sensitive cells. An overview of what is known of the pathways in amino acid-sensitive ORNs of Xenopus laevis tadpoles is shown in Fig. 1.1.

After successful transduction and transformation of an odor signal, action potentials travel via ORN axons to glomeruli that form the first odor representation in the brain. The numerous ORNs projecting to the same glomerulus are thought to have the same olfactory receptor(s) and therefore the same odorant response profiles. Studies investigating ORNs that have either identical receptors or react to the same stimulus revealed the existence of both concentration-dependent and -independent firing (Rospars et al., 2000, Martelli et al., 2013, Grosmaitre et al., 2006, Connelly et al., 2013, Rospars et al., 2013). Those results suggest differences in intraglomerular activity patterns and are described in more detail below.

Rospars et al. (2013) studied ORN populations that react to the same stimulus. Dosedependent activation revealed different thresholds and distinct dynamic ranges between ORNs. This is consistent with studies investigating ORNs that had the same olfactory receptor (Grosmaitre et al., 2006, Connelly et al., 2013). However, the maximal firing rate and the median number of spikes across ORN populations was surprisingly constant, representing the concentration-independent firing (Rospars et al., 2013). Studies 


\section{Introduction}

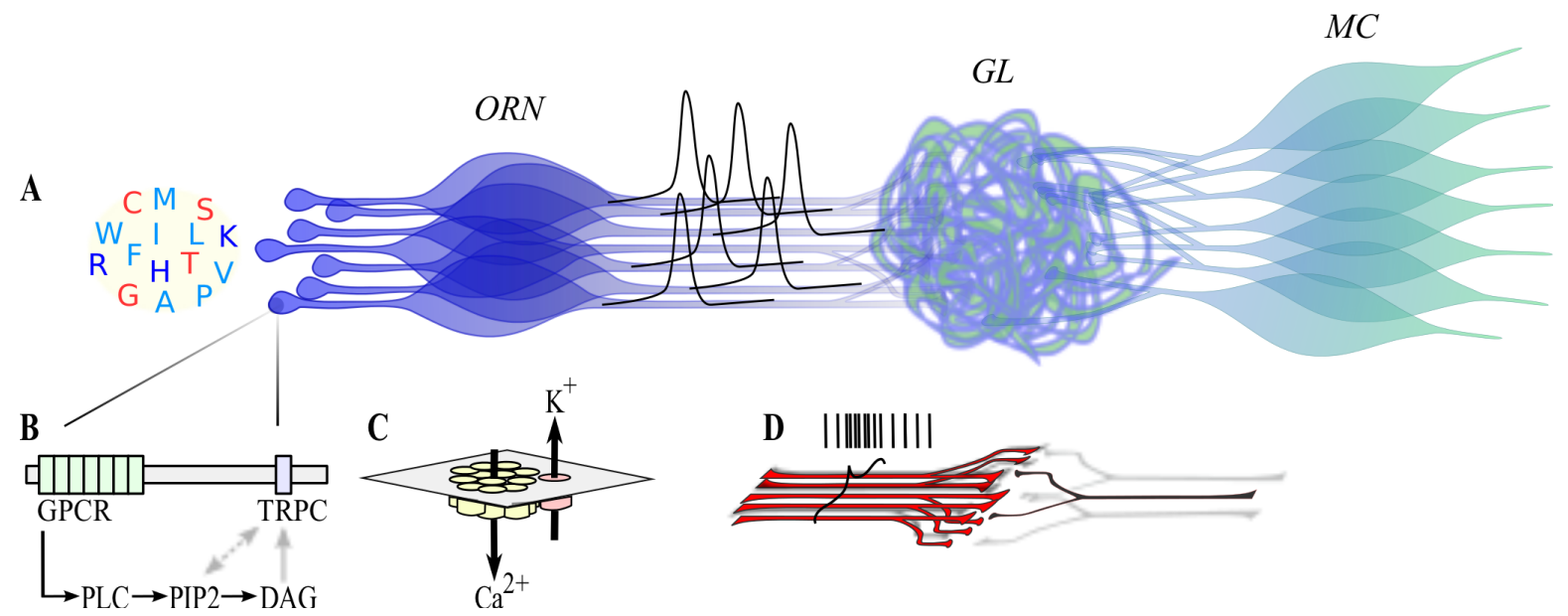

Figure 1.1: Amino acid-sensitive cells of Xenopus laevis tadpoles.

A: Stimulated amino acid-sensitive ORNs generate action potentials. They are conducted via ORN axons to glomeruli (GL) which are located in the olfactory bulb. The presynaptic odor code is subsequently converted into a post-synaptic signal in mitral cells (MC). B: Amino acid-sensitive ORNs are endowed with the PLC-mediated pathway. Stimulusinduced activation of PLC, via GPCRs, results in the cleaving of PIP2 and probably leads to a direct and/or indirect activation of TRPCs. C: The shape of amino acid-induced action potentials, and thereby the sensitivity of those ORNs, is determined by the functioning of HVA (yellow) and BK channels (red). D: Branching axons of multiple ORNs contribute to the final presynaptic odor representation within and across glomeruli. ORN: olfactory receptor neuron; PLC: phospholipase C; GPCRs: G-protein coupled receptors; PIP2: phosphoinositide; TRPC transient receptor potential channel; HVA: high voltage activated $\mathrm{Ca}^{2+}$ channel; BK: $\left[\mathrm{Ca}^{2+}\right]$-activated large-conductance potassium channel.

focusing on glomerular activity patterns have repeatedly shown that an increased odor concentration results in the recruitment of more glomeruli (e.g. Wachowiak and Cohen, 2001 (calcium imaging) and Meister and Bonhoeffer, 2001 (intrinsic signals). An increase in the number of activated glomeruli changes the raw glomerular odor map. Surprisingly, the quality or identity of an odor representation is retained (Cleland et al., 2007). The authors namely showed that a normalization of the complete glomerular map shows a constant pattern throughout different concentrations. A mechanism possibly underlying an increase of activated glomeruli, but a preserved odor quality, could be an increase of activated intraglomerular ORNs. If this was the case, ORNs projecting to one glomerulus could have different response thresholds. Since spontaneous neuronal 


\section{Introduction}

firing is correlated with neuronal sensitivity, spontaneous activity could be different across intraglomerular ORNs as well. The results obtained by Connelly et al. (2013) support this hypothesis. They reported that spontaneous activity patterns are different in distinct ORN populations, but do not correlated with near-saturation-induced responses (Connelly et al., 2013). Surprisingly, there is still a rather prominent variability within ORN populations having the same receptor (Connelly et al., 2013).

Although focusing on ORN subpopulations deepens our understanding of odor processing, it gives limited insight into single glomerular processing. One limitation is that ORNs having the same receptor do not necessarily project to the same glomerulus. In rodents they project to at least two glomeruli per hemisphere. In marmosets (Moriya-Ito et al., 2015) and whales (Kishida et al., 2015), both mammals, there is evidence that ORN populations even project to multiple glomeruli. Another limitation is that most ORN population studies have been performed at the level of the olfactory epithelium, thereby probably neglecting processes occurring in ORN axon terminals (e.g. GABA-mediated adaptation; Murmu et al., 2011 (Drosophila), Wachowiak et al., 2005 (mice)). The importance of those processes is illustrated by Wachowiak et al. (2004). They suggest that intraglomerular activity patterns cannot be functionally distinguished. The above results underline that it is essential to investigate intraglomerular activity patterns at the level of the olfactory bulb to get a better understanding of presynaptic intraglomerular activity patterns. 


\section{Introduction}

\subsection{Goal of the Thesis}

The main goal of the experiments presented in this thesis was, to get more insight into presynaptic intraglomerular response profiles and spontaneous activity patterns. In addition, I investigated how the activity of individual intraglomerular axons can be separated.

Xenopus laevis tadpoles were used as an animal model. Differences in ORN response patterns were to be expected in those animals, because tadpole ORNs have broad response profiles (Manzini and Schild, 2004). Secondly, as the same study suggests that tadpole ORNs might have more than one functional odorant receptor, adaptation processes might be more distinct across ORNs of this species. Thirdly, the axons of ORNs branch (Hassenklöver and Manzini, 2013) and project to multiple distinct glomeruli (Nezlin and Schild, 2005), a feature that might lead to a higher diversity in presynaptic ORN response profiles of $X$. laevis.

To study presynaptic intraglomerular activity patterns I used fast fluorescent 3D calcium imaging and activity correlation imaging. These methods have been suitable approaches for studying odor coding in the brain (Junek et al., 2009, Junek et al., 2010). Since glomeruli are dense neuropils, a reliable discrimination of activity patterns requires the proper selection of intraglomerular regions of interest. To investigate what type of ROI selection is the most suitable for studying activity patterns within single glomeruli, three different ROI selection procedures were tested on experimental data. Furthermore, simulated data was used to compare semi-automatically and automatically detected ROIs.

To obtain a better understanding of the presynaptic odor representation within single glomeruli, dose-response profiles, odorant response profiles and spontaneous activity patterns were compared. Furthermore, I investigated which conditions are required to distinguish individual intraglomerular axons based on activity patterns alone. 


\section{Methods}

\subsection{Sample preparation}

\subsubsection{Electroporation}

To visualize ORN axon terminals, Xenopus laevis tadpoles (stage 52-54, Nieuwkoop and Faber, 1994) were electroporated, as previously reported in Bao et al., 2015. In short, we used $0.02 \%$ MS-222 (ethyl 3-aminobenzoate methanesulfonate; Sigma-Aldrich) to anesthetize the tadpoles (Hassenklöver and Manzini, 2014). Small parts of calcium green-1 dextran (CaGreen; MW: 3000 Da, Sigma-Aldrich) or JG205 crystals were placed into both nostrils. For optimal electroporation of the lateral cluster one electrode was positioned in the nostril and the other electrode on the skin between the nostril and the eyes (see Fig. 2.1). In the case of CaGreen three pulses (20 ms, $20 \mathrm{~V}, 1 \mathrm{~Hz}$ ) were applied. Then the electrodes were slightly repositioned (by moving them up and down), and three additional pulses were delivered (see Fig. 2.1A). The same procedure was performed for the other nostril. JG205 electroporation differed by the pulse settings only. Six hundred pulses ( $1 \mathrm{~ms}, 15 \mathrm{~V})$ were given at $200 \mathrm{~Hz}$. As a control staining, some of the animals were electroporated with JG205 and Alexa 680 simultaneously. After the electroporation, the animals were housed in the dark at room temperature. Food and fresh water were provided regularly. Following a recovery period of at least 2 days, the animals were sacrificed. Experimental procedures were conducted according to the guidelines of the Göttingen University Committee for Ethics in Animal Experimenta- 


\section{Methods}

tion.

\subsubsection{Slicing and whole mount preparation}

Tadpoles were chilled in a mixture of water and ice and killed by decapitation. A tissue block which contained the olfactory mucosae, olfactory nerves and olfactory bulb was cut out and subsequently used for slicing (Manzini et al., 2002a) or for whole mount preparation (Hassenklöver and Manzini, 2013). During the slicing procedure, the tissue block was glued on the specimen plate and the dorsal part of the olfactory bulb was removed using a vibratome (VT1200S, Leica). To complete the slice a second cut, which was as ventral as possible, was performed. The sample was then transferred into a small recording dish filled with Ringer's solution (see chapter 2.2). The tissue was stabilized by a net that was made of platinum wire and nylon threads.

Whole mount preparation was achieved by performing the following steps (see Fig. 2.1). The tissue block was cut out and positioned on a preparation dish with the ventral side of the tissue block facing up (Fig. 2.1D). Pins inserted between the olfactory nerves were used to provide stability. A few drops of Ringer's solution (section 2.2) were added to prevent dehydration. Next, a pair of scissors was used to remove the meninges covering the ventral part of the brain (Fig. 2.1D). After preparation, the tissue was transferred to a recording chamber filled with Ringer's solution and the sample was stabilized with a net, as described above. As shown in Fig. 2.1D, the threads of the net covered neither the epithelium nor the anterior part of the olfactory bulb. In this way stimuli can be applied and glomerular imaging can be performed (see sections 2.3 and 2.4). 


\section{Methods}
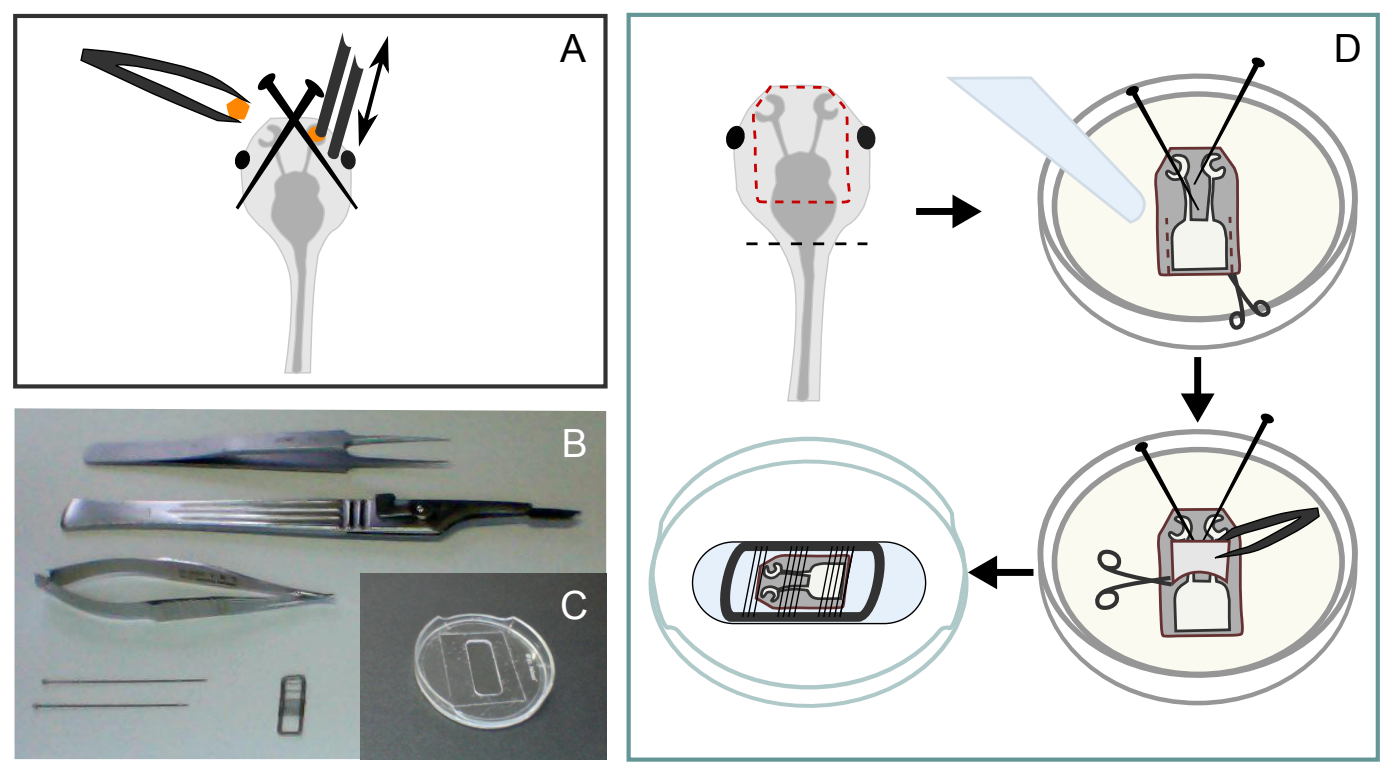

Figure 2.1: Sample preparation.

A: Electroporation of ORNs. B: Instruments used for whole mount preparation, see D. C: Recording chamber. D: Black dashed line: spinal cord cut. Red dashed lines: tissue block. Blue dashed lines: brain covering tissue that is removed. After exposing the brain, the sample was transferred to a recording chamber and stabilized with a net.

\subsection{Solutions and stimuli}

The composition of the Ringer's solution was (in mM): $98 \mathrm{NaCl}, 2 \mathrm{KCl}, 1 \mathrm{CaCl}_{2}$, $2 \mathrm{MgCl}_{2}, 5$ glucose, 5 sodium pyruvate, 10 Hepes. Furthermore the osmolarity was adjusted to $225-235 \mathrm{mOsm} / \mathrm{l}$ and the $\mathrm{pH}$ to 7.8 . Fifteen single amino acids or a mixture of them were used to stimulate olfactory receptor neurons (Manzini et al., 2002b, sections 2.3 and 2.4), namely, L-alanine, L-arginine, L-cysteine, glycine, L-histidine, L-isoleucine, L-leucine, L-lysine, L-methionine, L-phenylalanine, L-proline, L-serine, L-threonine, L-tryptophan, L-valine (Sigma-Aldrich). Fresh or frozen stock solutions $(10 \mathrm{mM})$ were diluted to the right concentration and were kept in the fridge. Solutions were used for no longer than one week. 


\section{Methods}

\subsection{Imaging of target glomeruli}

Volume stacks of images were obtained using a custom built line illumination microscope (by Alexander Brinkmann). Excitation was achieved using a Sapphire Laser (488nm; Coherent). As CaGreen and JG205 have different properties, e.g. basal intensity and bleaching properties, the imaging settings were changed according to the dye used. First the lateral cluster, which is a morphological and functional distinct group of glomeruli (Manzini et al., 2007b, Gliem et al., 2013), was located using fluorescence. Then a two- or three-dimensional time recording of the lateral cluster was obtained $(512 * 512(* 5)$ pixels, 30-60 s, min. $2 \mathrm{~Hz})$. Synchronously, a mixture of amino acids (10 or $100 \mu \mathrm{M}$; section 2.2) was administered via a funnel, which was positioned closely to the olfactory epithelium (see also Gliem et al., 2013). The first images were used to spot the location of possible target glomeruli. The voxel size of images taken after this initial experimental phase was always smaller than $1 \mu \mathrm{m}^{3}$. As a next step, a detailed z-stack was obtained for regions that responded to amino acid application. A z-stack consisted of at least 20 consecutive z-planes (distance $1 \mu \mathrm{m}$ ). If this z-stack revealed a spherical neuropil, the latter structure was considered as an anatomical and functional glomerulus of the lateral cluster. 


\section{Methods}

\subsection{Experimental conditions}

\subsubsection{Stimulus-induced responses}

Repeated stimulation and dose-response profiles have revealed differences within ORN subpopulations as reported in former studies (Rospars et al., 2013, Grosmaitre et al., 2006, Connelly et al., 2013). I repeated these approaches at the level of the olfactory bulb as follows. After the selection of a target glomerulus (section 2.3) an amino acid mixture (AA-mix) containing 15 amino acids was applied at four different concentrations, namely $500 \mathrm{nM}, 1 \mu \mathrm{M}, 10 \mu \mathrm{M}$ and $100 \mu \mathrm{M}$. Subsequently, one concentration was chosen for repetitive application. In order to exclude floor or ceiling effects the selected concentration should show a clear but not saturated response. After obtaining a spontaneous activity recording, the solution was applied 10 times using an interstimulus interval of 20 seconds. Since a control stimulus (Ringer's solution) was applied as the first stimulus, a full recording consisted of 11 applications in total (see Fig. 2.2). If imaging conditions allowed more recordings, this measurement was repeated. Repetitive application of a single amino acid was performed in a similar manner. As a threshold concentration for single amino acids I used $1 \mu \mathrm{M}$ (Breunig et al., 2010).

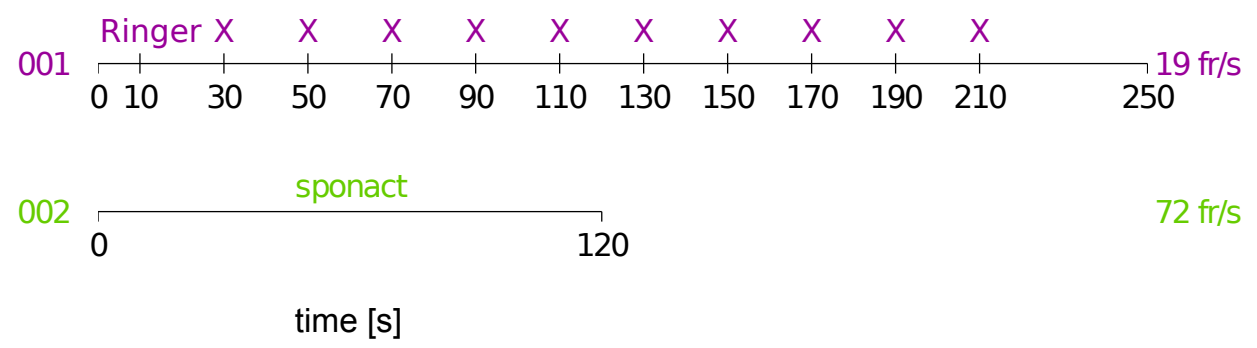

Figure 2.2: Experimental design of repetitive odor stimulation.

Each recording started with a Ringer's solution application. The odor stimulus (X), which was determined before, is then applied ten times with an interstimulus interval of 20 seconds. Spontaneous activity was obtained in a second measurement. 


\section{Methods}

The selection of the most suitable single amino acid was achieved as follows. Fifteen amino acids (10 $\mu \mathrm{M}$, see section 2.2) were applied consecutively.

Bleaching and movement of the tissue are rather prominent in long measurements, which is a disadvantage when applying all amino acids in one recording. On the other hand, to facilitate the choice of the optimal stimulus, as many stimuli as possible should be applied within one trial. For these reasons the different amino acids, at a concentration of $10 \mu \mathrm{M}$ each, were divided over three subsequent recordings (see Fig. 2.3). When single amino acids did not show a response it was either because they they did not activate the glomerulus, or the ORNs projecting to this glomerulus had died. In order to exclude the latter, AA-mix $[10 \mu \mathrm{M}]$ was applied after the application of the $5^{\text {th }}$ and $15^{\text {th }}$ amino acid. Furthermore, to exclude that changes in fluorescence were induced by the application itself instead of the odorant, Ringer's solution was applied in the beginning of the second recording (Fig. 2.3).

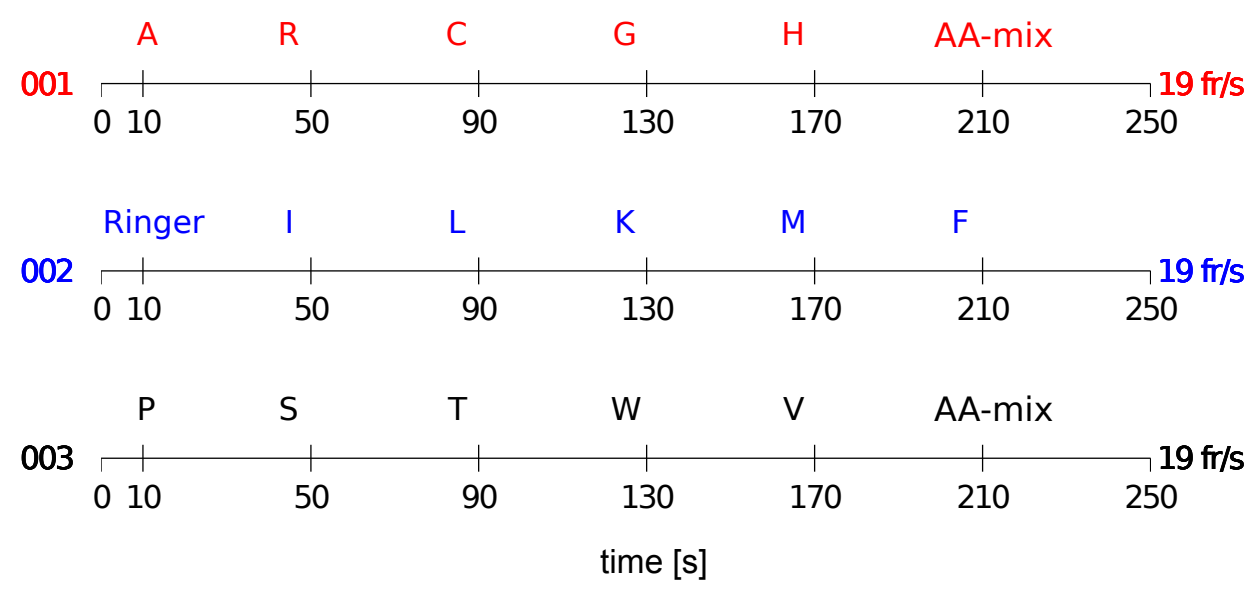

Figure 2.3: Experimental design of single amino acid stimulation.

During each recording five amino acids and one control stimulus (either amino-acid mixture or Ringer's solution) were applied. The application order of single amino acids varied across experiments. 


\section{Methods}

Dose-response profiles were obtained in a third set of physiological experiments. Different concentrations of the AA-mix in the range $40 \mathrm{nM}$ to $100 \mu \mathrm{M}$ were applied. Similarly to the previous conditions, Ringer's solution was applied before administering other stimuli (see Fig. 2.4). This trial was either followed by a recording of spontaneous activity or the recording was repeated.

When using stimuli, volume stacks of images of CaGreen-stained glomeruli were recorded using the following imaging conditions: volume stacks of images (10 consecutive imaging planes) were acquired for ca. $4 \mathrm{~min}$. at a frequency of 1.9 stacks/s. The distance between consecutive z-planes was either 1 or $2 \mu \mathrm{m}$. During experiments there was a continuous Ringer's solution flow $(250 \mathrm{ml} / \mathrm{hour})$. The above described imaging settings were not suitable for JG205-stained glomeruli. Strong bleaching was observed in the initial seconds of the first recording and stimulus-induced responses were only occasionally observed. To reduce bleaching, the laser intensity was reduced so that responses could only be recognized after the recording when studying ROI traces. Furthermore, a recovery time of at least one week was required to observe responses to amino acids in JG205-stained glomeruli (see section 3.4).

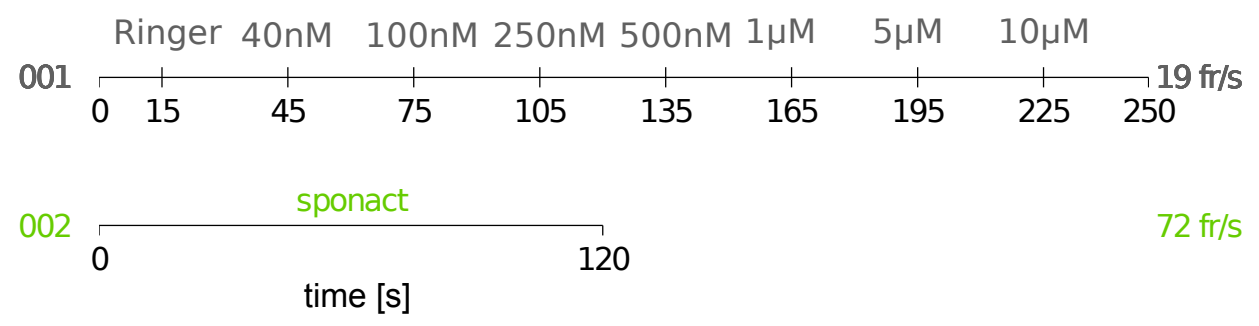

Figure 2.4: Experimental design of dose-response experiments.

Every dose-response recording started with a Ringer's solution application. Different concentrations of the amino acid mixture were then applied using an interstimulus interval of 30 seconds. Spontaneous activity was obtained in a second measurement. 


\section{Methods}

\subsubsection{Spontaneous activity}

Spontaneous $\mathrm{Ca}^{2+}$ fluctuations were usually difficult to discriminate from background noise. In this thesis, a spontaneous activity recording refers to a recording without amino-acid applications. Spontaneous activity recordings of CaGreen stained ORNs were generally obtained using an imaging frame rate of $7.2 \mathrm{~Hz}$ to avoid under sampling. Lower frame rates (min. $3.8 \mathrm{~Hz}$ ) were only used if the fluorescence signal was low. An imaging rate of $3.8 \mathrm{~Hz}$ turned out to be still sufficient for obtaining the shortest events measured in fast $(40 \mathrm{~Hz}) 2 \mathrm{D}$ images of ORNs stained with CaGreen (see also section 3.3). Spontaneous activity patterns were compared between ORNs stained with CaGreen or with JG205. Since ORNs stained with JG205 were rather prone to bleaching a reduced recording frame rate was used to visualize spontaneous activity over longer time $(1.9 \mathrm{~Hz})$.

\subsection{Image processing}

In 4D measurements, slight movements of the slice were observed in all directions. Bleaching occurred depending on the recording time or dye used. Furthermore, fixed lines with different intensities were observed in the recorded images. Matlab scripts (written by Dr. G. Bao and Dr. M. Alevra) were used to correct for those phenomena (Bao and Schild, 2014; Alevra, 2013 (PhD thesis)). First, to correct for the stripes, the image was
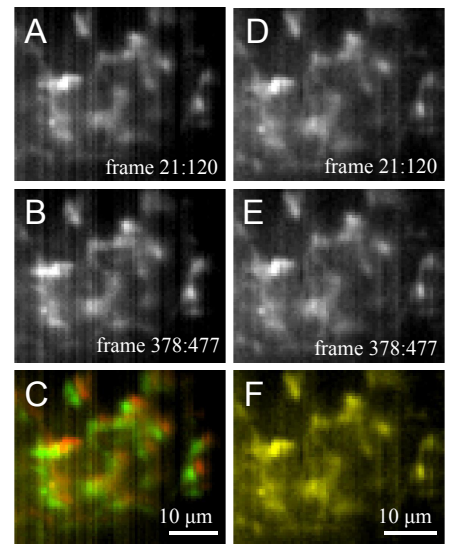

Figure 2.5: Movement correction. Glomerular position in the beginning and in the end of a typical CaGreen recording (A, B). C: Superposition of images A and B. D-F: A-C after movement correction. 


\section{Methods}

averaged in the same direction as the stripes. As a next step, this mean stripe was correlated with the image. The correlation value was then multiplied with the average stripe and subsequently subtracted from the input image. (see Fig. 2.6).

Secondly, to correct for movement a reference frame was defined. Further, the maximum shift (in pixels) was estimated by the experimenter and this number was zeropadded to all frames. Fast Fourier transform was used to correlate the reference frame with all other frames individually. The resulting shift vector was corrected for border effects and pixels were back shifted using cubic spline interpolation (Fig. 2.5, Alevra, 2013 (PhD thesis)). Legendre low pass filters were used to correct for bleaching (Bao and Schild, 2014, Fig. 2.6).

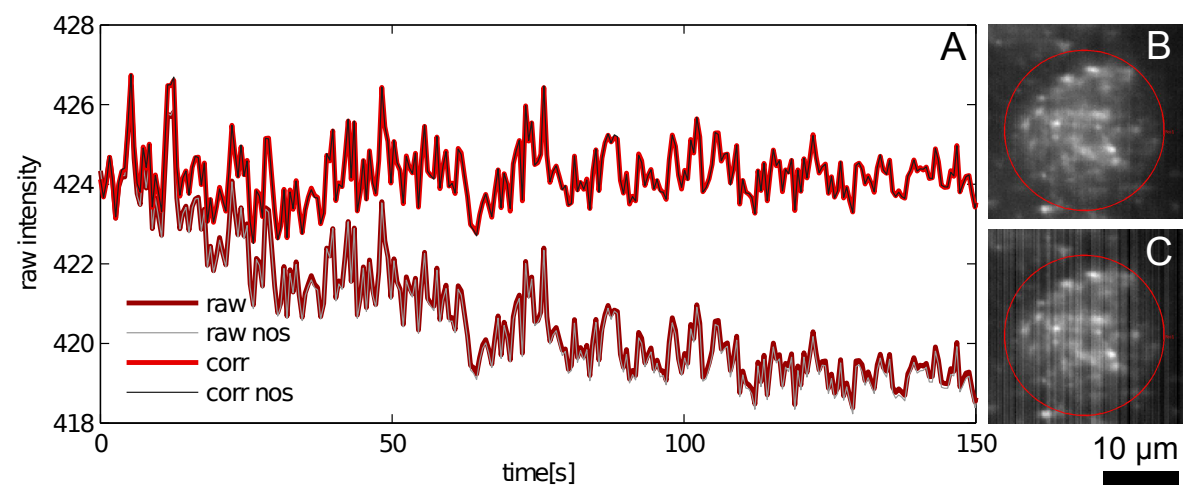

Figure 2.6: Bleach and line correction.

A: Average traces corresponding to the ROI indicated in B and C (red line). B: corrected and time averaged z-plane of a glomerulus stained with JG205. C: Similar to B, now before correction. raw: no Legendre low pass filter; raw nos: no Legendre low pass filter, stripes were removed (no stripes); corr: Legendre low pass-filtered image; corr nos: Legendre low pass-filtered image, stripes are removed. 


\section{Methods}

\subsection{Selection of regions of interest}

The second goal of the thesis was to distinguish intraglomerular axons. Proper ROI selection is essential for this purpose - mainly, because ROIs usually contain more than one voxel. ROI size or a slight different ROI position can therefore influence its average signal or trace significantly. As for the axon density within glomeruli, proper ROI selection could be crucial when using activity-based differentiation of intraglomerular compartments. In this study three different ways of selecting ROIs were compared.

\subsubsection{Manual ROI selection}

Manual selection was accomplished by drawing 2D lines around intraglomerular structures. ROIs covered the same glomerular area in all repetitions. In other words structures that appeared or disappeared over multiple recordings, due to z-movement or bleaching, respectively, were disregarded.

\subsubsection{Semi-automatic ROI selection}

Semi-automatic ROI selection was performed as described in Junek et al., 2009 and Chen et al., 2009, using a custom written software called aciPeel (by Dr. M. Alevra). First, a 3D neighborhood correlation map of the input image was generated (Fig. 2.7AB). In this greyscale map, pixel values corresponded to correlation. Then 3D ROIs were generated surrounding a manually chosen pixel (Fig. 2.7C). The ROI diameter (x, y, z) was set independently. The traces, which corresponded to the mean of its pixels, were used as reference traces for activity correlation imaging (Junek et al., 2009, (Fig. 2.7D)). ACI correlates the reference trace with the traces of all other pixels for each ROI. Each correlation map gets assigned another color. If the traces are clearly distinct this method can visualize distinct morphological structures with different activity patterns. 

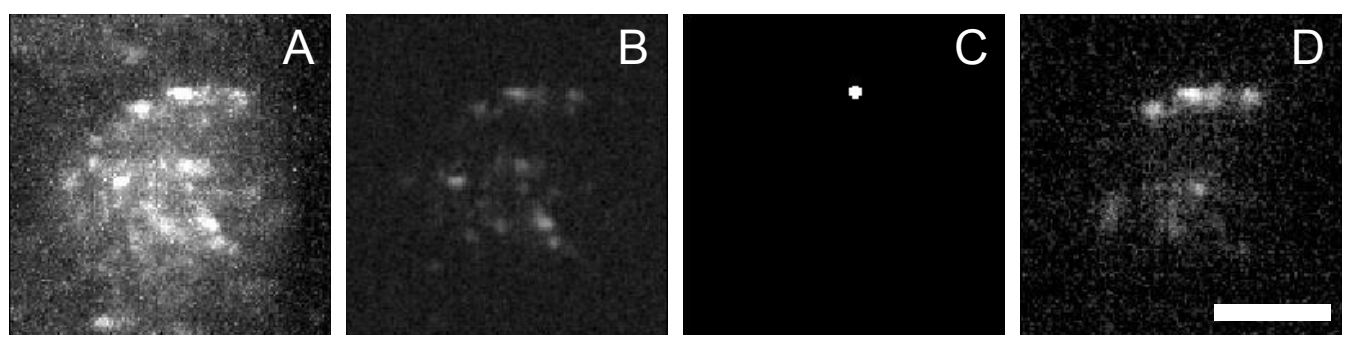

Figure 2.7: Semi-automatic ROI selection.

A: One example z-plane of a glomerulus stained with JG205 (maximal projection in time). B: Neighborhood correlation map of A. C: Drawn ROI. D: Activity correlation map of ROI trace. Scale bar $=10 \mu \mathrm{m}$.

\subsubsection{Automatic ROI selection}

Spontaneous activity patterns were assumed to be typical for each intraglomerular axon. Therefore, automatic ROI selection was accomplished by a voxel-wise analysis of spontaneous fluorescent fluctuations (script written by Prof. Dr. Dr. D. Schild). The time traces of a voxel block (min. $1 \mu \mathrm{m}^{3}$ ) were averaged over space (equation 2.1 ) and time, and the filtered trace was assigned to its central voxel (Fig. 2.8).

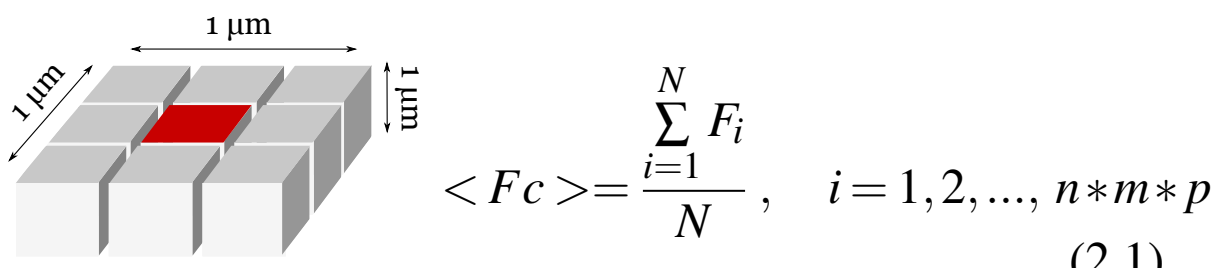

Figure 2.8: Spatiotemporalfilter.

Time traces of a voxel block are filtered over space (equation 2.1) and time and subsequently assigned to the central voxel of this block. $\mathbf{n}^{*} \mathbf{m} * \mathbf{p} *$ : the total number of averaged voxels in space $(\mathrm{x}, \mathrm{y}, \mathrm{z})$ 


\section{Methods}

After filtering, an event threshold was set. Fluorescence intensities above this threshold were defined as initial events and their corresponding time points (or frames) were saved. Thus, each voxel had its own sequence of event-related time points. Voxels that had different sequences were regarded as different ROIs. Hence, it is crucial to define which sequences belong to the same ROI and which do not. Here, we used strong criteria which are described next.

First of all, all sequences were listed. Then sequences consisting of only one event were removed, in other words, those particular voxels were neglected. Since axons were assumed to have a diameter of at least $1 \mu \mathrm{m}$, sequences should be found in at least $n$ voxels, depending on voxel size. This lower limit of occurrences was therefore also the minimum number of voxels within one ROI. With an increasing value the number of ROIs will decline, thereby reducing the number of false positive ROIs, but increasing false negatives. The following steps will describe how voxels were added to the initial ROIs. Since there was a time difference of at least $100 \mathrm{~ms}$ (max. $500 \mathrm{~ms}$ ) between the first and the last imaged z-plane, sequences are low-pass filtered. Identical sequences were merged, in other words, those sequences were assigned to the same ROI. Then sequences were sorted by their event onset and correlated. Highly correlated (e.g. > .9) events were merged as well. Furthermore, if all events of one voxel were also found in the sequence of another voxel, the former was added to the ROI of the latter. In Fig. 2.9A an overview of detected events are shown and in Fig. 2.9B an example ROI is shown. Fig. 2.9C shows the thirteen event sequences that were detected in the automatically defined ROIs. 


\section{Methods}
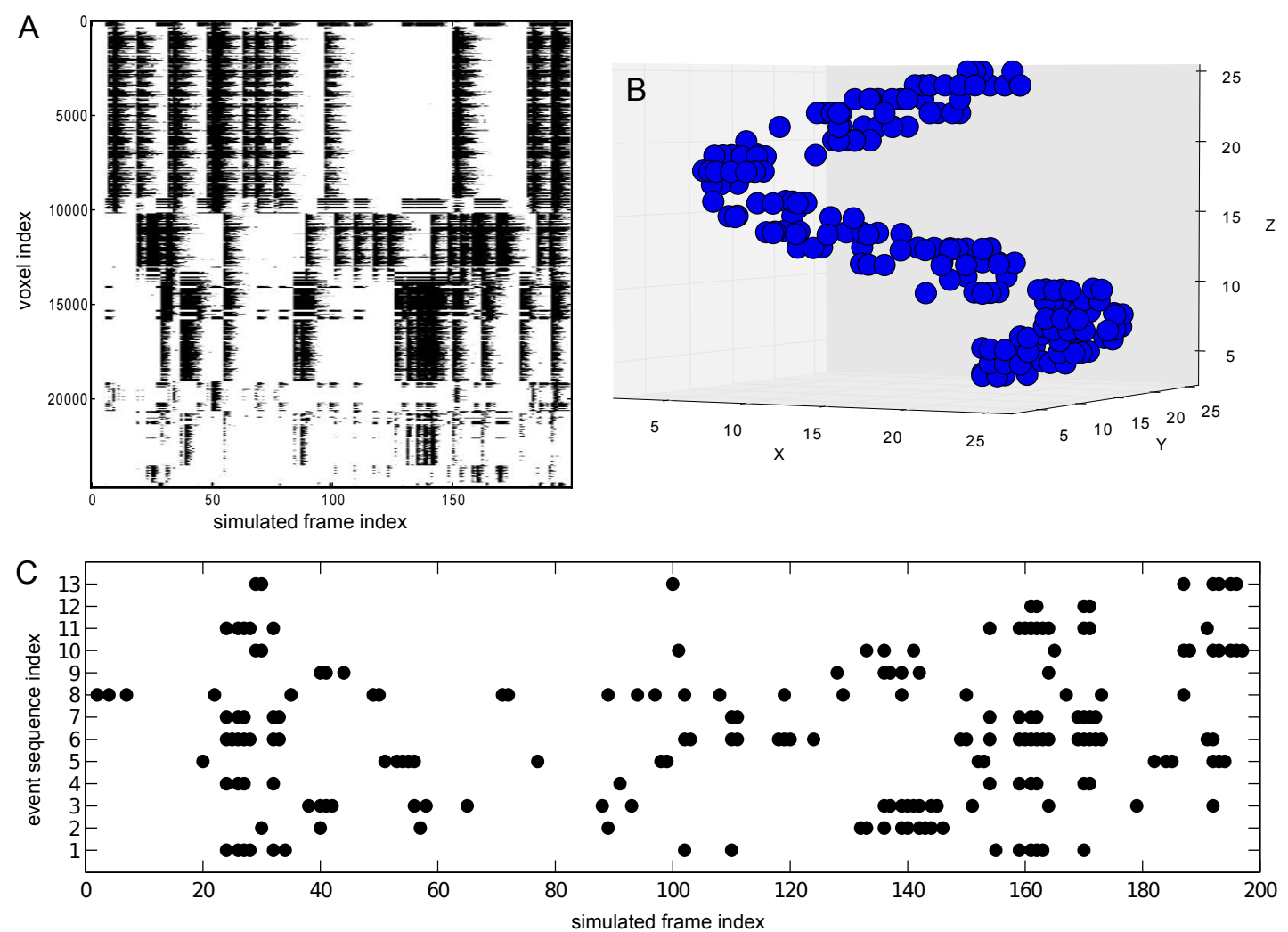

Figure 2.9: Automatic ROI detection.

A: Initial detected events for every voxel that contained more than one event. B: One of the thirteen detected ROIs. C: Event sequences detected in the automatically defined ROIs. 


\section{Methods}

\subsection{Response analysis}

For comparing intraglomerular activity patterns, $\Delta F / F_{0}$ values were taken. $\Delta F / F_{0}$ values were calculated according to the following equation, where $F_{0}$ corresponds to the average intensity of the initial five seconds of a recording:

$$
\Delta F / F_{0}=\frac{F-F_{0}}{F_{0}}
$$

Maximum responses were defined as follows. First, $\Delta F / F_{0}$ intensities were temporally filtered over three frames. Then the local maximum after trigger onset was detected. Each maximum was corrected for its pre-stimulus intensity, meaning the average intensity from five seconds before trigger onset.

To detect responses usually some kind of threshold is set based on the fluorescent intensities before stimulus onset. The observation that Ringer's solution application sometimes induced fluctuations in the fluorescent intensity revealed that this method was unsatisfactory for detecting responses. It is important to correct for this effect to avoid inaccurate detected responses (false positives). Here, response thresholds were defined by the sum of two background intensities. The first intensity was the maximum intensity over the time after Ringer's solution application and before the next application plus its standard deviation. The second intensity was the maximum intensity of 5 seconds before stimulus application plus its standard deviation. The sum of those two intensities was defined as the response threshold. Responses were detected whenever the maximum intensity after stimulus application was above the assigned threshold value. 


\section{Results}

\subsection{Dose-response profiles}

The presynaptic ORN fibers of the glomeruli were stained with either CaGreen or JG205. 3D recordings of stimulus-induced responses and spontaneous activity were obtained to investigate presynaptic intraglomerular activity patterns and glomerular sensitivity. Furthermore, I studied the activity patterns of axonal branches within single glomeruli and investigated how they can be assigned to different ORNs.
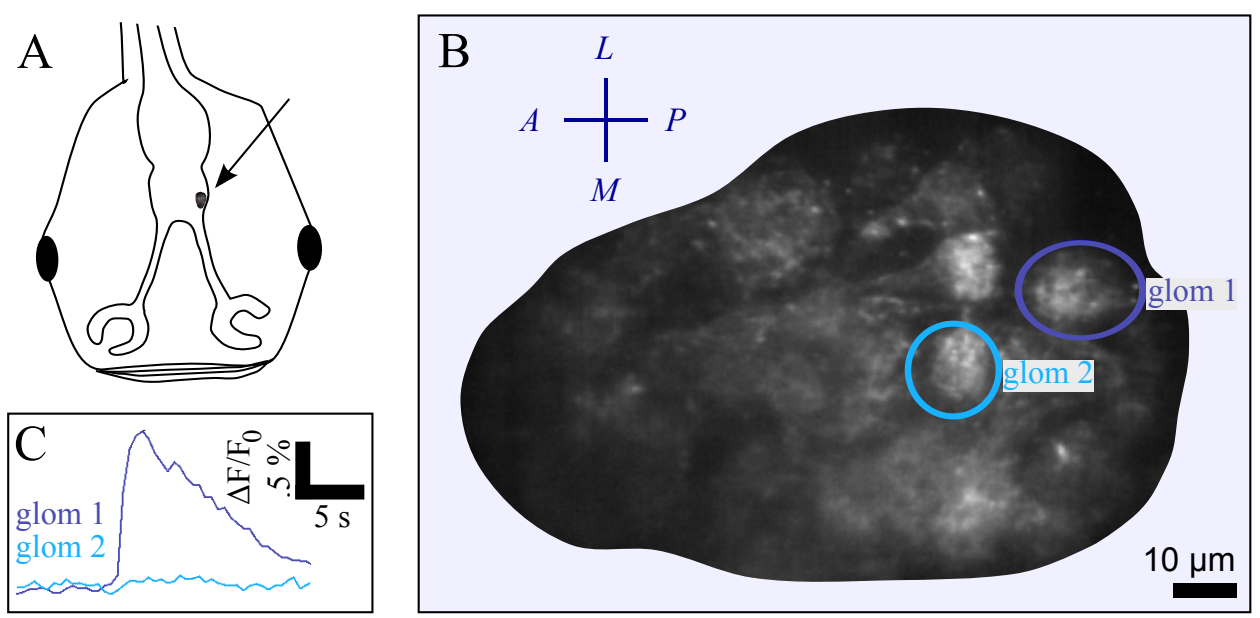

Figure 3.1: Selection of target glomeruli.

A: Schematic representation of a X. laevis tadpole head. The arrow indicates the position of the lateral olfactory bulb. B: Glomeruli within the lateral cluster stained with CaGreen. C: One glomerulus responded to stimulation with AA-mix $[10 \mu \mathrm{M}]$, the other did not. A: anterior; $P$ : posterior; $M$ : medial; $L$ : lateral. 


\section{Results}

Functional glomeruli of the lateral cluster were located using an amino-acid mixture of either 10 or $100 \mu \mathrm{M}$ (Fig. 3.1). Subsequently, dose response profiles were obtained using different AA-mix concentrations ranging from $40 \mathrm{nM}$ to $100 \mu \mathrm{M}$.

Half of the selected glomeruli were very sensitive, even reacting to odor stimuli in the nanomolar range (Table 3.1 and Table 3.2).

\begin{tabular}{|l|l|l|l|l|}
\hline & $c_{\min }$ & $R_{\min }$ & $R_{\max }$ & $c_{\max }$ \\
\hline glom 1 & $40 \mathrm{nM}$ & $40 \mathrm{nM}$ & $5 \mu \mathrm{M}$ & $5 \mu \mathrm{M}$ \\
\hline glom 2 & $40 \mathrm{nM}$ & $5 \mu \mathrm{M}$ & $5 \mu \mathrm{M}$ & $5 \mu \mathrm{M}$ \\
\hline glom 3 & $40 \mathrm{nM}$ & $500 \mathrm{nM}$ & $5 \mu \mathrm{M}$ & $5 \mu \mathrm{M}$ \\
\hline glom 4 & $40 \mathrm{nM}$ & $40 \mathrm{nM}$ & $250 \mathrm{nM}$ & $10 \mu \mathrm{M}$ \\
\hline glom 5 & $40 \mathrm{nM}$ & $250 \mathrm{nM}$ & $5 \mu \mathrm{M}$ & $10 \mu \mathrm{M}$ \\
\hline glom 6 & $40 \mathrm{nM}$ & $40 \mathrm{nM}$ & $1 \mu \mathrm{M}$ & $10 \mu \mathrm{M}$ \\
\hline glom 7 & $40 \mathrm{nM}$ & $5 \mu \mathrm{M}$ & $10 \mu \mathrm{M}$ & $10 \mu \mathrm{M}$ \\
\hline
\end{tabular}

Table 3.1: Glomerular response thresholds after stimulation with $10 \mu \mathrm{M}$ AA-mix.

Lowest used concentration $\left(c_{\min }\right)$. Threshold $\left(R_{\min }\right)$ and maximum responses $\left(R_{\max }\right)$. Maximal used concentration $c_{\max }$.

\begin{tabular}{|l|l|l|l|l|}
\hline & $c_{\min }$ & $R_{\min }$ & $R_{\max }$ & $c_{\max }$ \\
\hline glom 1 & $500 \mathrm{nM}$ & $10 \mu \mathrm{M}$ & $10 \mu \mathrm{M}$ & $100 \mu \mathrm{M}$ \\
\hline glom 2 & $500 \mathrm{nM}$ & $500 \mathrm{nM}$ & $100 \mu \mathrm{M}$ & $100 \mu \mathrm{M}$ \\
\hline glom 3 & $500 \mathrm{nM}$ & $10 \mu \mathrm{M}$ & $100 \mu \mathrm{M}$ & $100 \mu \mathrm{M}$ \\
\hline glom 4 & $500 \mathrm{nM}$ & $10 \mu \mathrm{M}$ & $100 \mu \mathrm{M}$ & $100 \mu \mathrm{M}$ \\
\hline glom 5 & $500 \mathrm{nM}$ & $500 \mathrm{nM}$ & $10 \mu \mathrm{M}$ & $100 \mu \mathrm{M}$ \\
\hline glom 6 & $500 \mathrm{nM}$ & $1 \mu \mathrm{M}$ & $100 \mu \mathrm{M}$ & $100 \mu \mathrm{M}$ \\
\hline glom 7 & $50 \mathrm{nM}$ & $10 \mu \mathrm{M}$ & $100 \mu \mathrm{M}$ & $100 \mu \mathrm{M}$ \\
\hline
\end{tabular}

Table 3.2: Glomerular response thresholds after stimulation with $100 \boldsymbol{\mu M}$ AA-mix. Lowest used concentration $\left(c_{\min }\right)$. Threshold $\left(R_{\min }\right)$ and maximum responses $\left(R_{\max }\right)$. Maximal used concentration $c_{\max }$. 


\section{Results}

To investigate whether intraglomerular activity patterns differed, spontaneous and stimulusinduced activity from small intraglomerular ROIs were compared ( $n=6$, Fig. 3.2 A-B). Within single glomeruli both response profiles and spontaneous activity patterns differed among some intraglomerular ROIs ( $n=25$, Fig. 3.2 C-D).
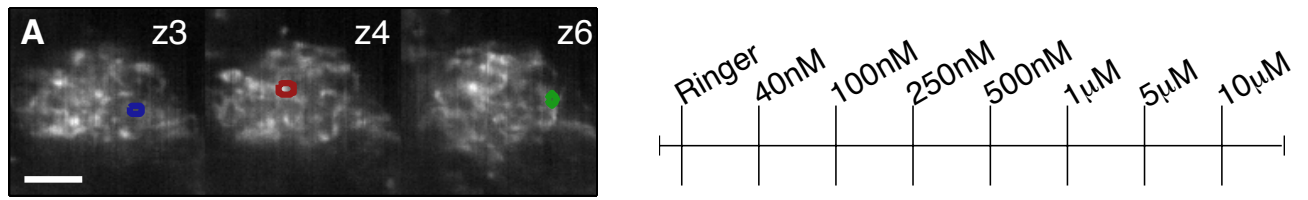

B
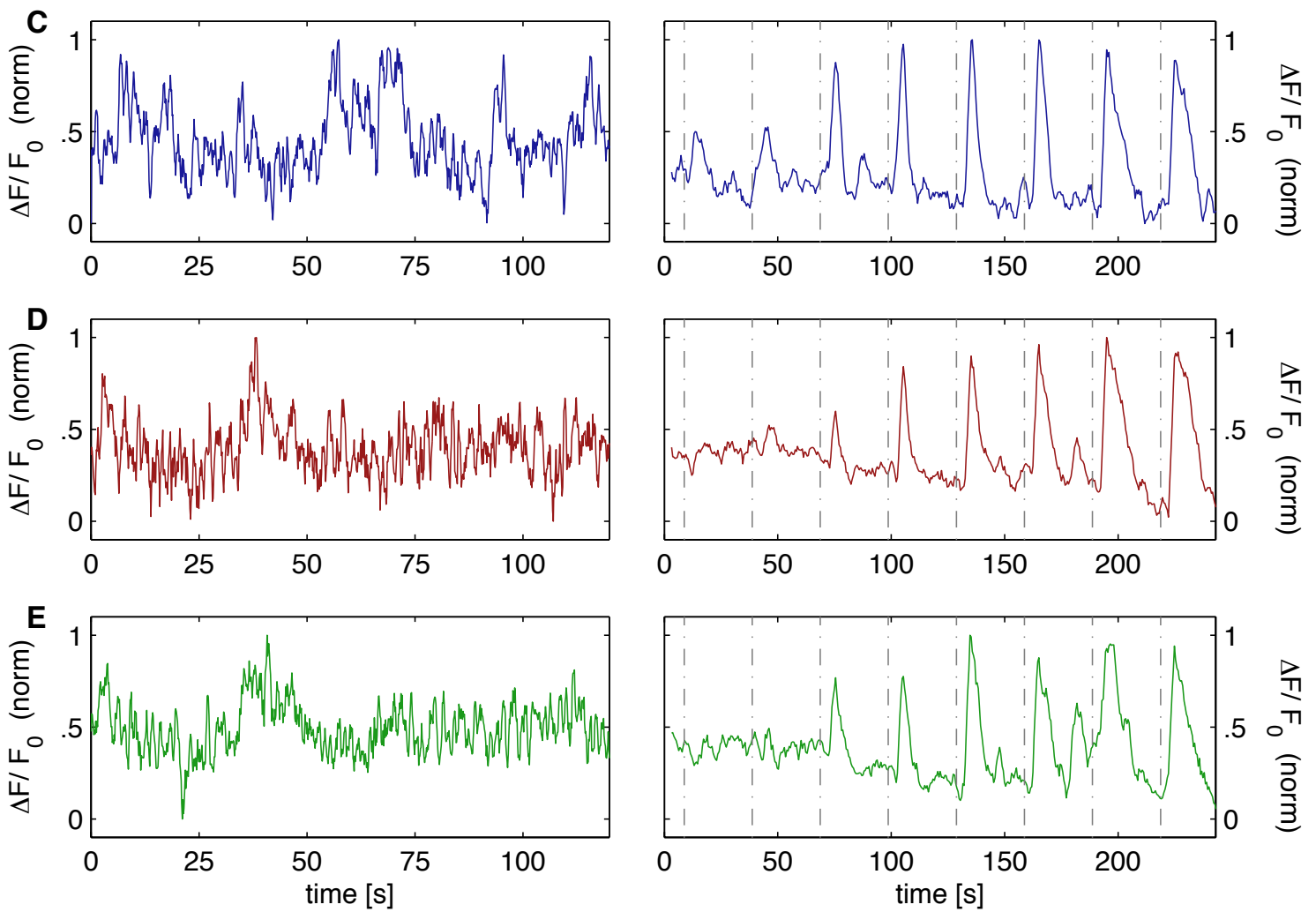

Figure 3.2: Differences between intraglomerular dose-response profiles and spontaneous activity patterns.

A: A Glomerulus. Three consecutive z-planes and one ROI in each plane. B: Stimulus application scheme. C-E. Spontaneous activity patterns (left) and dose-response profiles (right) corresponding to the ROIs indicated in A. Scale bar $=10 \mu \mathrm{m}$. 


\section{Results}

However, ROIs with seemingly different response amplitude profiles (Fig. 3.2 C-E, right) sometimes had spontaneous events that overlapped in time (Fig. 3.2 C-E, left). The spontaneous traces show moreover, that $\mathrm{Ca}^{2+}$ fluctuations can be very long-lasting and usually have a low frequency. (Fig. 3.2 C-E, left).

Previously, correlation between activity patterns has been used to visualize populations of mitral cells and their dendrites (Junek et al., 2009), which form the post-synaptic side of glomeruli. Here, I tested whether this method is suitable to discriminate individual axons within single glomeruli.

At the level of mitral cell activity, correlation imaging (ACI) was optimal when using spontaneous activity. ROIs were usually selected with the help of a neighborhood correlation map. The neighborhood correlation map of CaGreen-stained ORNs revealed a very poor correlation between neighboring pixels (Fig. 3.3), despite the differences in spontaneous activity found using manual ROI selection (Fig. 3.2C-D, left). Indeed correlated spontaneous events were observed only occasionally in CaGreen-stained ORN axons. Differences in dose response profiles were small (Fig. 3.2C-D, right). Therefore ACI maps were almost identical for all ROIs, and they revealed the whole glomerular structure instead of individual axons.

Figure 3.3: Neighborhood correlation of spontaneous activity.

A glomerulus (A) and its neighborhood correlation map (B). Scale bar $=10 \mu \mathrm{m}$.
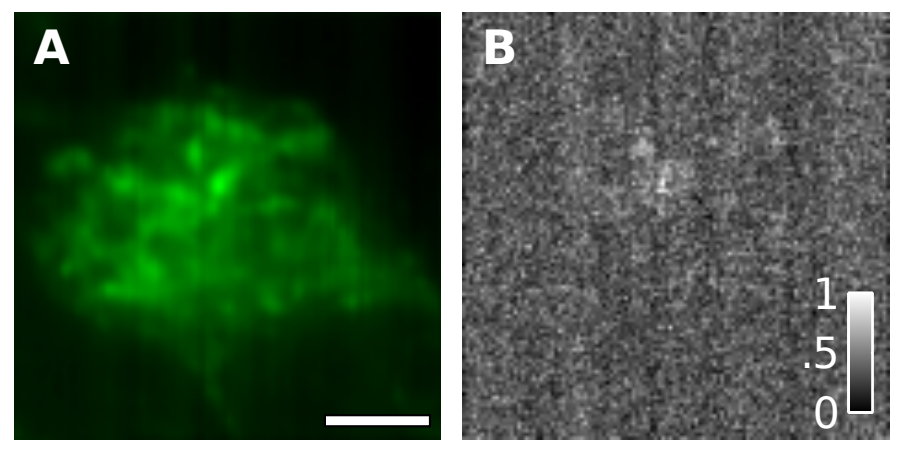


\section{Results}

In order to properly distinguish individual axons within single glomeruli, correlated events need to be axon-specific and, at the same time, those events should occur regularly. Is it possible to obtain cell-specific activity which does not require short and frequent spontaneous events? Is an activity-based visualization of intraglomerular axons possible without using ACI?

The dose-response experiments revealed differences in response profiles between intraglomerular regions. The strongest fluctuations were found after application of low concentrations of odorants (Fig. 3.2C-D). To investigate whether response thresholds are a reliable criterium for separating intraglomerular axons, dose-response experiments were repeated and response thresholds were compared over trials $(n=6)$.

In Fig. 3.4 the traces from three repetitions are shown. Activity patterns after the application of low concentrated stimuli looked quite different over repetitions. On the other hand, stimulation with AA-mix $[100 \mu \mathrm{M}]$ resulted in similar responses over subsequent recordings (Fig. 3.4A-C). It was therefore investigated, whether the activity differences observed after the application of low concentrated stimuli depend on previous applications with high concentrated stimuli. Response thresholds were compared over subsequent recordings of the same glomerulus, and between target glomeruli that were selected after the stimulation with either 10 or $100 \mu \mathrm{M}$ AA-mix. Over subsequent recordings, increased, decreased or unchanged response thresholds were observed. Furthermore, response thresholds did not differ between target glomeruli selected with $10 \mu \mathrm{M}(n=7)$ or those selected with $100 \mu \mathrm{M}$ AA-mix $(n=7$, student's t-test, $p=0.06$, Fig. 3.4E). Other observations show, that, response detection based on intensity changes might be limited for threshold concentrations, which is revealed by the traces of the second recording shown in Fig. 3.4B. After the application of $10 \mu \mathrm{M}$ AA-mix, around $t=170 \mathrm{~s}$, there was namely still an event that resembled a response (rectangle in Fig. 3.4B). 


\section{Results}

Focusing on the similarities between the activity patterns of the blue and red ROI over repetitions, revealed that most of the events were correlated. These ROIs could therefore cover different areas of the same axon.Interestingly, some events in the blue trace do not appear in the red trace (arrows in Fig. 3.4C). If those ROIs belonged to the same axon, which was likely based on the number of synchronous events, uncorrelated events occurred within the same axon.

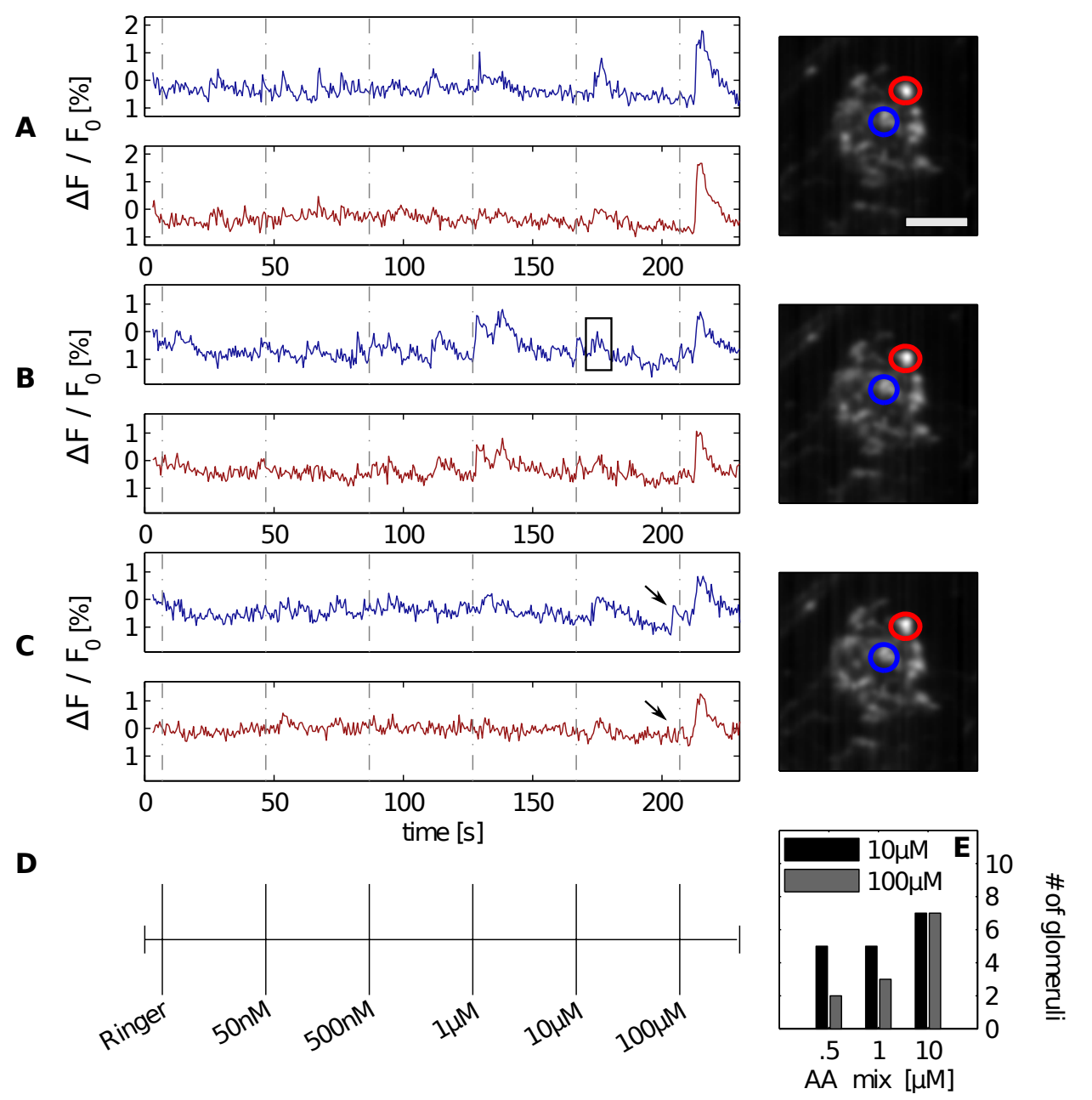

Figure 3.4: Differences and similarities over subsequent trials.

A-C: Maximal projections of an example glomerulus and its $\Delta F / F_{0}$ traces over three consecutive recordings. D: Stimulus application scheme. E: Thresholds of target glomeruli selected after 100 or $10 \mu \mathrm{M}$ AA-mix application. Scale bar $=10 \mu \mathrm{m}$. 


\section{Results}

The results presented so far show that spontaneous activity and response profiles are different among intraglomerular regions. The second goal of this thesis was to separate individual axons based on activity patterns. I investigated spontaneous activity and stimulus-induced patterns to test whether they are suitable for the visualization of individual axons within glomeruli. However, as presented before, spontaneous events were too rare and stimulus-induced responses too alike for a reliable visualization of individual axon branches (Fig. 3.2 and Fig. 3.4).

This underlines that frequent and correlated cell-specific activity is required to reliably distinguish individual axons based on activity patterns. The results from repeated dose-response experiments confirmed that the majority of the events (spontaneous or stimulus-induced) should have a detectable amplitude and that uncorrelated events within one axon cannot be excluded.

Nevertheless, repetitions of dose-response profiles showed that on the one hand, the shape of stimulus-induced responses to low concentrations fluctuates over repetitions. At the same time, correlated activity patterns from different ROIs also correlated in the subsequent recordings. Increasing the number of repetitions over a shorter time span would hypothetically not influence the number of uncorrelated events but increase the number of correlated events. The interstimulus interval can be lowered when response fluctuations are short. Since responses to low AA-mix concentrations are shorter than those induced by high AA-mix concentrations (Fig. 3.2), the repetitive application of low-concentrated stimuli is a suitable approach for eliciting short responsess. Furthermore, as mentioned earlier, fluctuations were most apparent at response threshold concentrations and those fluctuations may be used to separate axons.

A disadvantage of using stimuli is that correlated action potentials among axons are to be expected. On the other hand, the response fluctuations observed after the application of low concentrated AA-mix might be cell-specific leading to cell-specific activity profiles. Also, stimulus-induced responses have most likely an amplitude which can be 


\section{Results}

detected in most areas of one axon, reducing the influence of method-dependent artifacts. Therefore, as a next step, repetitive applications of a low AA-mix concentration were applied.

\subsection{Fluctuations of stimulus-induced responses}

As described above, stimulation with low AA-mix concentrations probably induces correlated activity among axons, but enough repetitions may result in a cell-specific response pattern. AA-mix threshold concentrations were defined as follows. First a doseresponse recording was obtained. Directly after recording uncorrected measurements were inspected. The lowest AA-mix concentration that induced a response throughout the glomerulus was chosen as the response threshold concentration. Then glomeruli were repeatedly stimulated with the chosen AA-mix concentration (Fig. 3.5B). Spontaneous activity patterns were recorded as well and served as a backup indication for different axons (Fig. 3.5C). In every single ROI responses were elicited repetitively during three identical measurements. (Fig. 3.5B, E, F).

As shown in Fig. 3.5, stimulus-induced responses were very similar and only occasionally clear differences between response profiles of different ROIs were observed (Fig. 3.5B). Another phenomenon observed was the general amplitude reduction over repetitions (Fig. 3.5B, E, F). The amplitude reduction was stronger for the red ROI than for the blue one. Response amplitudes might correlate with basal $\mathrm{Ca}^{2+}$ levels. Bleaching for example could lead to less available dye and therefore a reduced response amplitude. Since basal $\mathrm{Ca}^{2+}$ levels always fluctuate over recordings, I compared the pre-stimulus basal $\mathrm{Ca}^{2+}$ levels of those ROIs with the response amplitudes. This analysis revealed that the observed amplitude reduction could not be explained by basal $\mathrm{Ca}^{2+}$ levels alone (Fig. 3.5D), although its contribution could not be excluded. 


\section{Results}
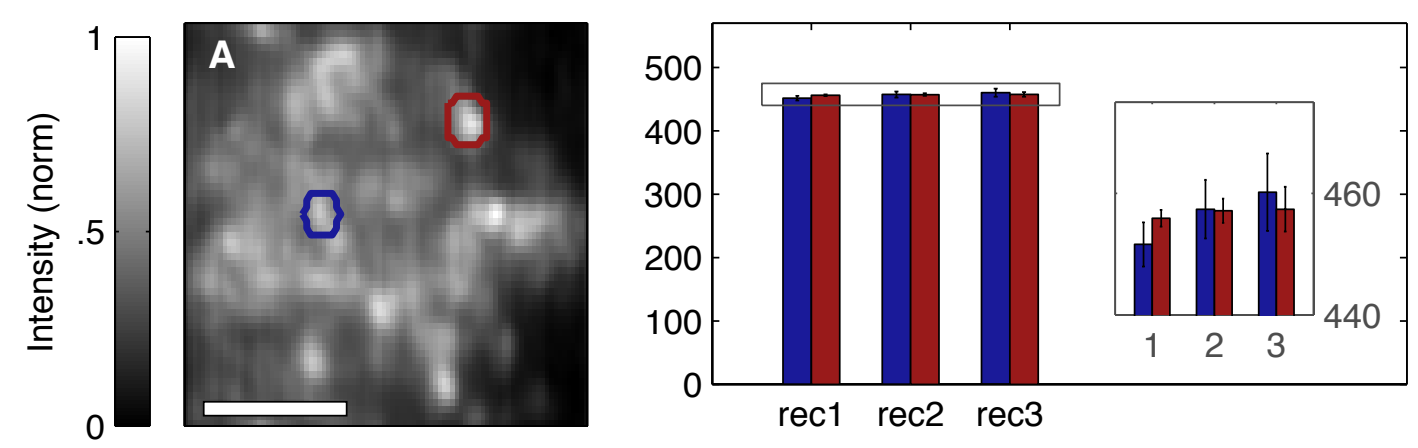

D
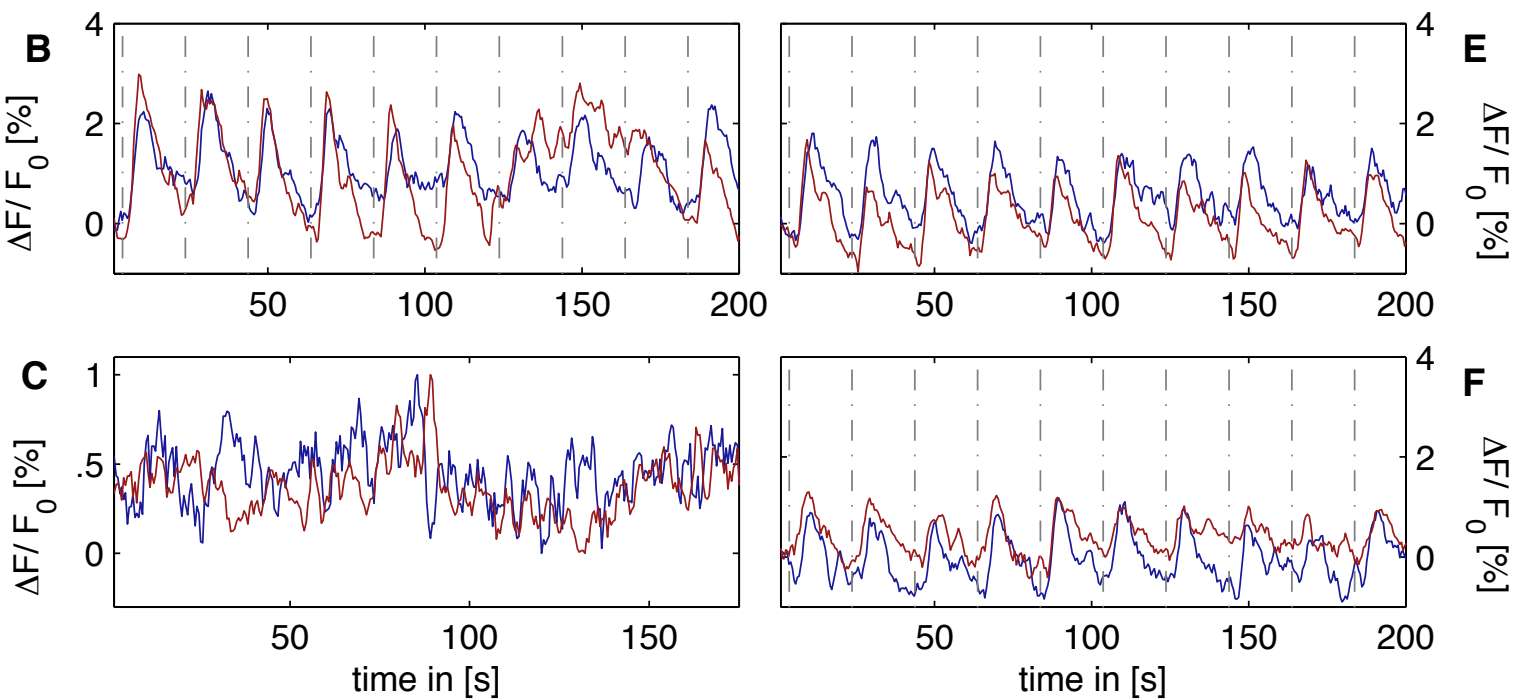

Figure 3.5: Repetitive stimulation reveals highly similar response profiles.

A: Maximal projection (time and $\mathrm{z}$ ) of a glomerulus. Its intensity is normalized to the maximal fluorescent intensity. Red and blue circles point out two ROIs used for data shown in B-F. Scale bar $=10 \mu \mathrm{m}$. B: Example of stimulus-induced $\mathrm{Ca}^{2+}$ fluctuations. Dashed lines indicate trigger onset. Small differences are present between the two ROIs. C: spontaneous activity recording revealing different spontaneous activity between the two ROIs. D: basal $\mathrm{Ca}^{2+}$ level fluctuations of three subsequent recordings. Bar colors correspond to the ROIs in A. E-F:. Repeated recording of B. 


\section{Results}

Despite the general amplitude reduction, responses were elicited repetitively. The small fluctuations between stimulus-induced responses were surprising, especially because spontaneous activity patterns were different. Could this effect be specific for AAmixtures? As a control experiment, single amino acids were repetitively applied $(n=5)$.
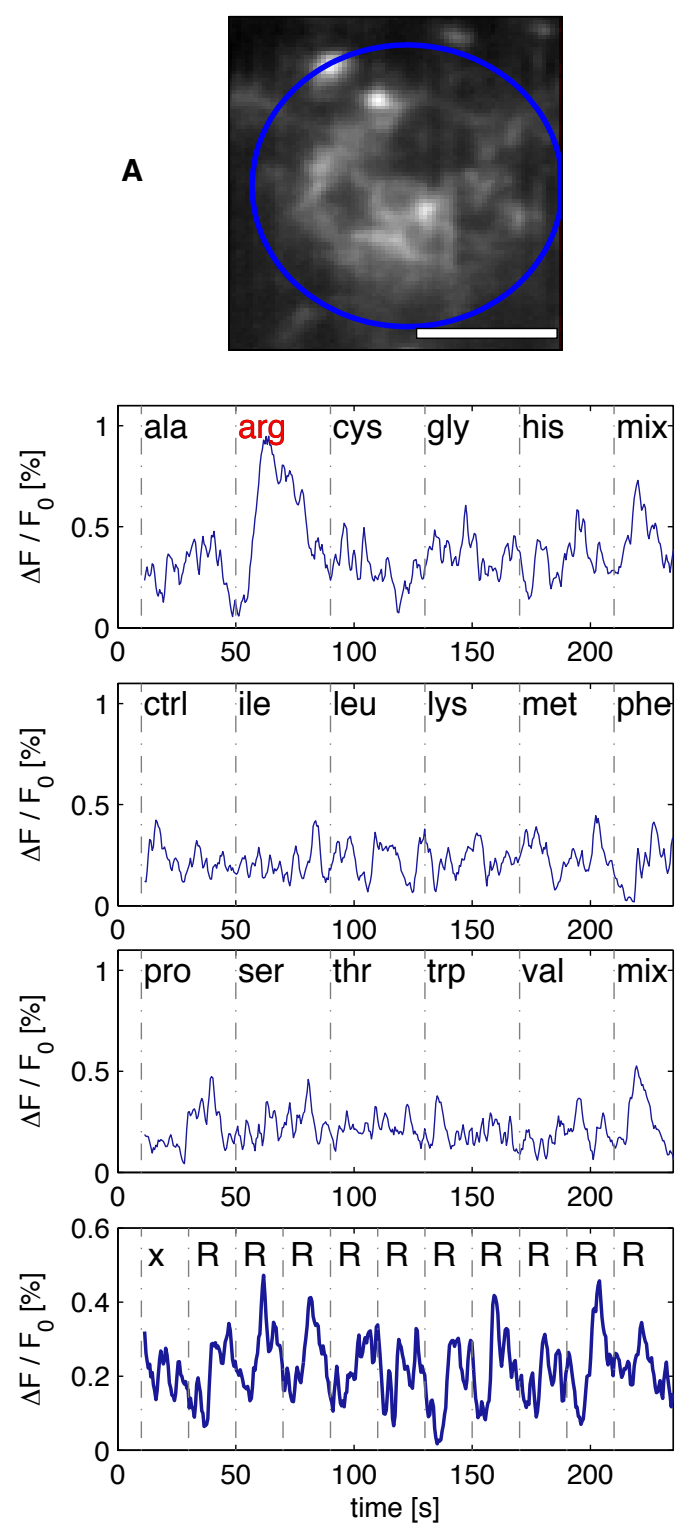

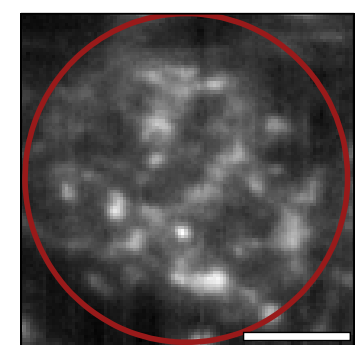

B
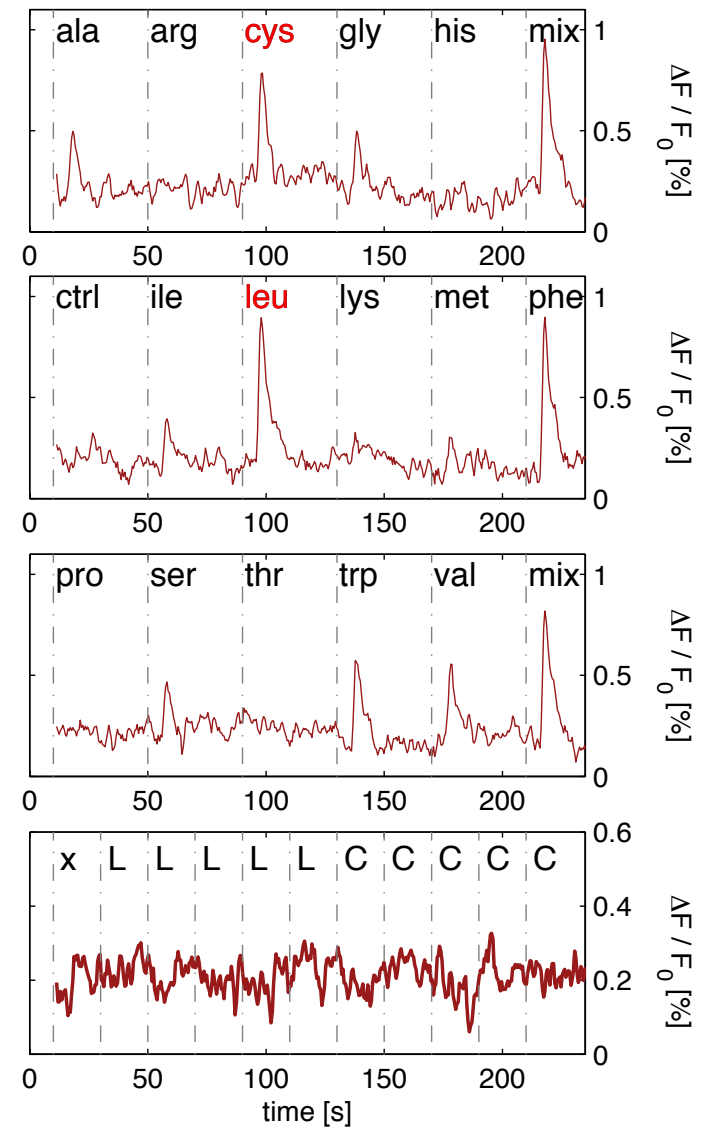

Figure 3.6: Two example glomeruli with different odorant response profiles.

A: A glomerulus responding to low concentrated arginine. B: A glomerulus not responding to low concentrated cysteine or leucine. Scale bar $=10 \mu \mathrm{m}$. 


\section{Results}

To select a suitable amino acid for repetitive stimulation, 15 different amino acids were subsequently administered before the repetitive stimulation procedure (see section 2.2, Fig. 3.7). Consequently, glomerular odor response profiles were obtained as well (Fig. 3.6 and Fig. 3.7).
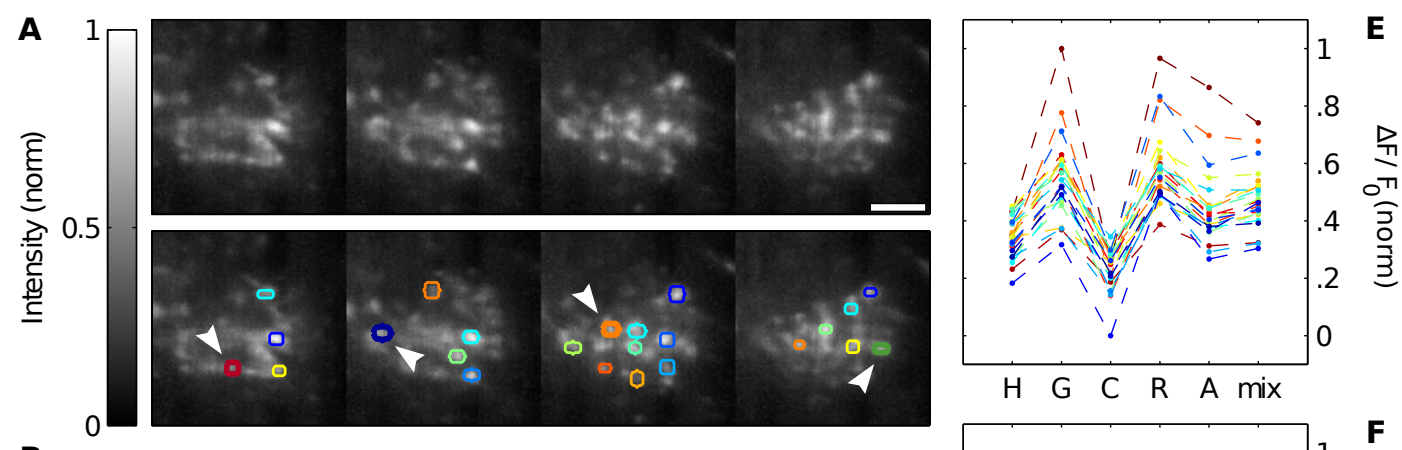

B
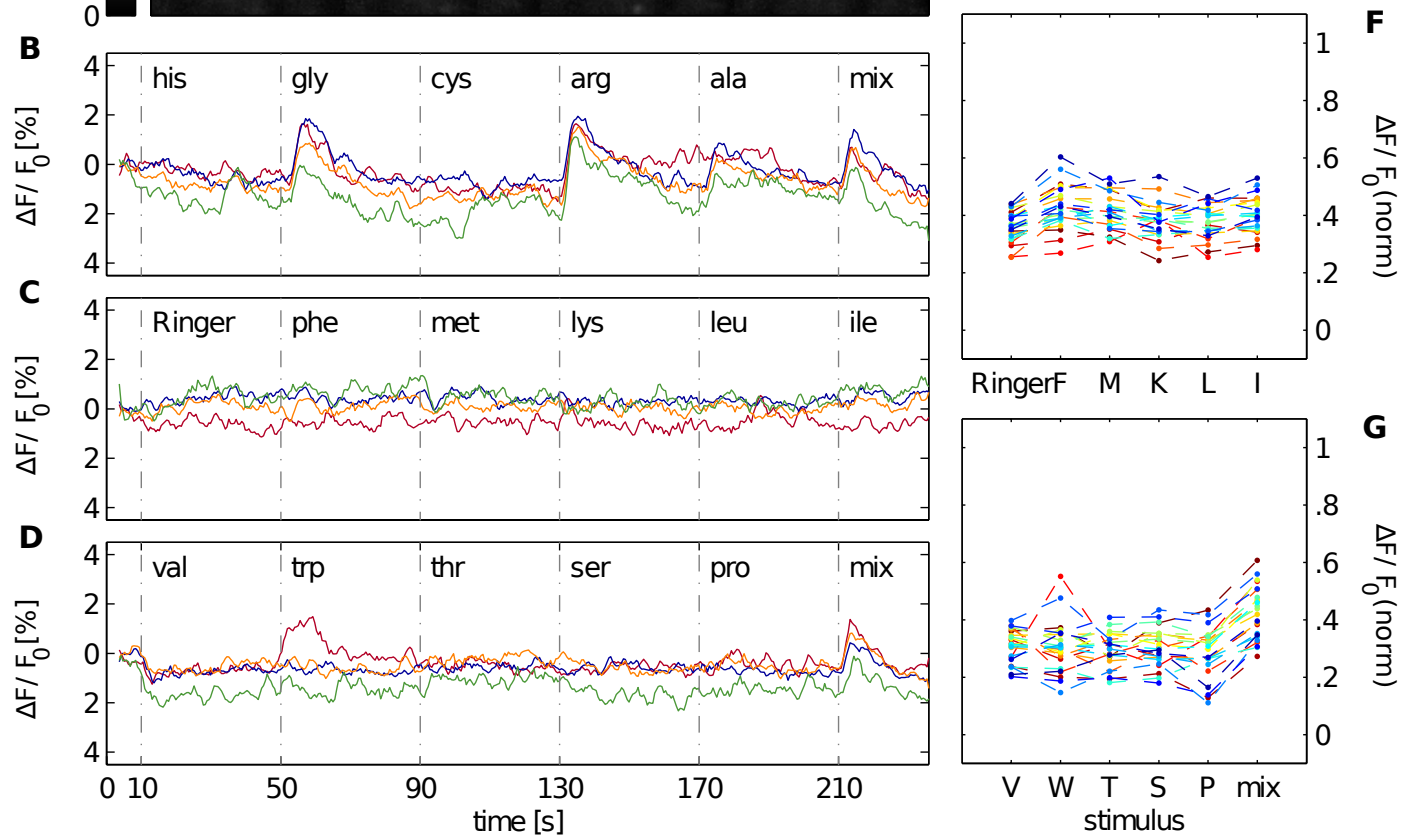

Figure 3.7: Intraglomerular odorant response profiles are very similar.

A: Maximal projection (in time) of four consecutive z-planes (upper row) and manually drawn ROIs (lower row). Scale bar $=10 \mu \mathrm{m}$. B-D: Odorant response profiles of four example ROIs, indicated by arrows in A. Three letter abbreviations are used for applied single amino acids. E-G: $\Delta \mathrm{F} / \mathrm{F}$ maxima (within $20 \mathrm{~s}$ after application) of all ROIs. 


\section{Results}

Before and after the repetitive stimulation with single amino acids a control experiment was performed to check whether the glomerulus was still able to respond. The condition of the slice was either validated by the application of an AA-mix $(10$ or $100 \mu \mathrm{M})$ or the repetition of the previous measurement. Five glomeruli only responded to the stimuli used in this control experiment. Three of them reacted to repetitive application of the chosen single amino acid (one example shown in Fig. 3.6 and one in Fig. 3.7). Interestingly, the odorant response profile of the glomerulus that did not react to the repetitive application of the chosen single amino acids was broader than the glomeruli that did react (Fig. 3.6).

To investigate whether response profiles differed among intraglomerular regions, the activity patterns of small ROIs $(n=24)$ were compared. Odorant response profiles turned out to be highly similar between regions (Fig. 3.7). For the example shown, the only ROI showing a somewhat different response pattern was located at the morphological border of this glomerulus (Fig. 3.7A, indicated in red).

Studying the response profiles from repetitive application revealed that it is complicated to distinguish reliably stimulus-induced odor responses from spontaneous fluctuations when using low concentrated single amino acids. The results represented in Fig. 3.8, suggest that arginine (R) induced responses fluctuate strongly within ROIs.

These results confirmed the hypothesis that responses induced by the repetitive application of low concentrated single amino acids will lead to strong fluctuations. However, the fluctuations within ROIs were sometimes bigger than the fluctuations between ROIs. When fluctuations within axons are bigger than among axons, it becomes very complex to reliably assign ROIs to axons. Moreover, the single-amino-acid experiments turned out to be very challenging and had a low success rate. Therefore, other experimental conditions were considered to distinguish intraglomerular axons based on activity profiles. 


\section{Results}
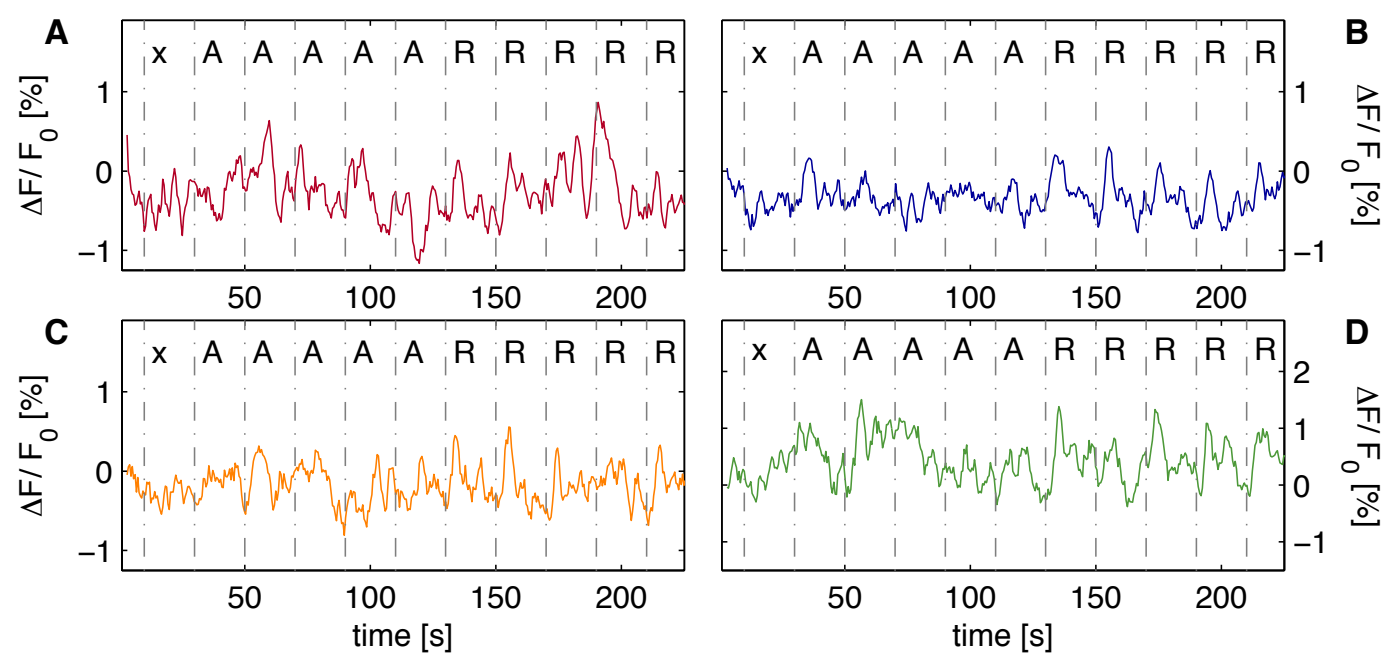

Figure 3.8: Strong fluctuations during repetitive stimulation with single amino acids A-D: Example traces of repetitive stimulation with single amino acids $(1 \mu \mathrm{M})$. Traces correspond to the ROIs indicated in Fig. 3.7. Single amino acids are indicated by single letter abbreviations and trigger onsets are indicated with dashed lines. Ringer's solution was applied during the first trigger $(\mathrm{x})$.

Previously, I observed that spontaneous activity was different among intraglomerular regions, although spontaneous events were rare. Obtaining more spontaneous activity would probably be a more effective approach than the categorization of stimulusinduced patterns. Since spontaneous bursts among axons can induce correlation between axons as well, the next sets of experiments focused on categorizing spontaneous activity. 


\section{Results}

\subsection{Different types of spontaneous activity}

Fig. 3.2 and Fig. 3.4 illustrate that different intraglomerular components showed different spontaneous events (Fig. 3.2C-E). Surprisingly, neighborhood correlation maps of spontaneous traces revealed only low correlation (see Fig. 3.3). This may be due to the low signal-to-noise ratio or to the relatively few but long spontaneous events that typically last more than 10 seconds (Fig. 3.2C-E). To validate whether the observed $\mathrm{Ca}^{2+}$ fluctuations were physiological, fast 2D measurements $(40 \mathrm{~Hz})$ were obtained using a laser scanning microscope (for an example see Fig. 3.9A). In these fast measurements, the fastest $\mathrm{Ca}^{2+}$ events from $\mathrm{CaGreen}$ stained glomeruli had a duration of at least 1 second (Fig. 3.9B). Longer events were also observed. The shape of the long events indicated that they reflect a summation of multiple short events.
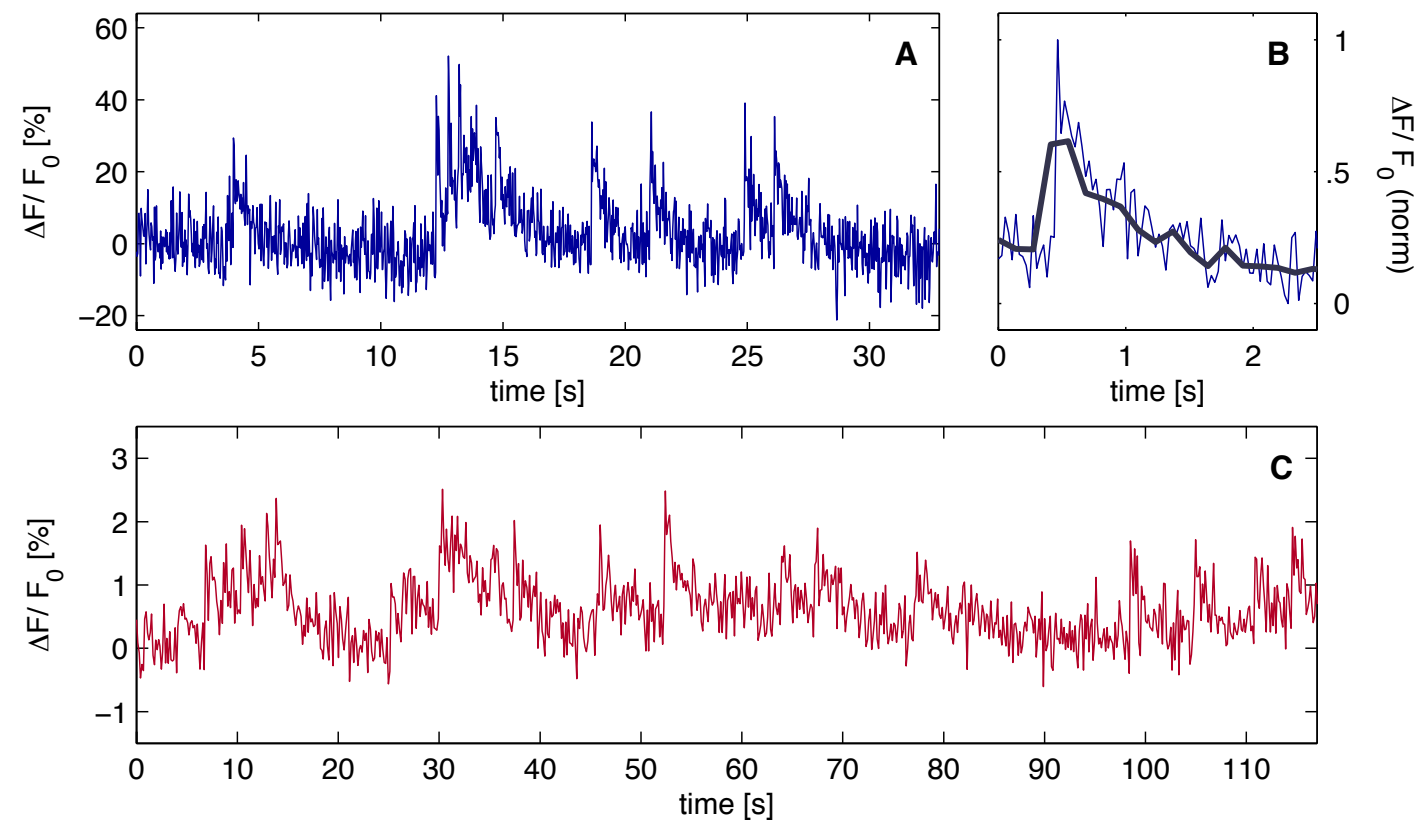

Figure 3.9: Spontaneous $\mathrm{Ca}^{2+}$ fluctuations

A: Example of 2D spontaneous activity $(40 \mathrm{~Hz}) \mathbf{B}$ : Mean $\mathrm{Ca}^{2+}$ spike (blue) from a $40 \mathrm{~Hz}$ recording and a simulated spike (black) using $3.8 \mathrm{~Hz}$. C: Example of 3D spontaneous activity $(3.8 \mathrm{~Hz})$. 


\section{Results}

\subsection{Presynaptic glomerular morphology and function}

Obviously, the summation of slightly different spike patterns could result in similarly long $\mathrm{Ca}^{2+}$ events. The fast 2D measurements already showed that the long events cannot be resolved by just faster imaging, because they are still observed when using imaging recording rates at $40 \mathrm{~Hz}$. As shown before, such events complicate reliable axon separation (Fig. 3.2). It could be that due to the dye dynamics of CaGreen, differences within similar long events cannot be detected. To reliably separate CaGreen-stained ORN axons, unphysiological conditions may be required. An easier approach is to use a dye with different $\mathrm{Ca}^{2+}$-binding dynamics. Recently, Julia Graf (Dept. of organic and biomolecular chemistry, Göttingen) developed a new calcium dye: JG205. Doseresponse profiles and spontaneous activity patterns of JG205-stained neurons were obtained and compared to CaGreen.
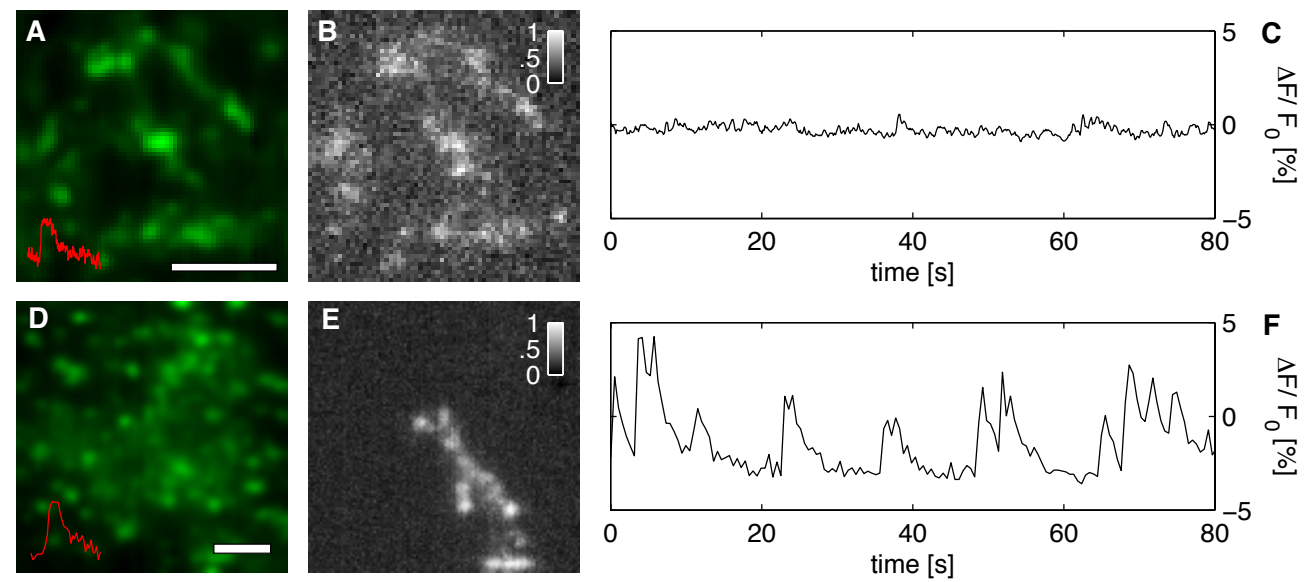

Figure 3.10: Signal-to-noise differences between different dyes.

A: Staining with Calcium Green-1 dextran, maximal projection over time and z. Red line: response to amino acid stimulation. B: The normalized activity correlation map reveals all pixels which are correlated with the trace shown in C. C: Spontaneous activity trace (CaGreen). D-F: Correspond to A-C and show results obtained with JG205. Both traces were recorded at $1.908 \mathrm{~Hz}$. Scale bar $=10 \mu \mathrm{m}$. 


\section{Results}

Activity patterns of JG205-stained ORNs had an improved signal-to-noise ratio compared to CaGreen-stained ORNs. (Fig. 3.10). Long events were still observed when using JG205, but the events now had a high characteristic profile (Fig. 3.10). Those activity patterns were used for generating ACI maps. As a next step, ACI maps were compared between recordings of CaGreen and JG205-stained ORNs. The maps clearly differed in quality, which became especially apparent after comparing them to the maximal projection of the obtained 4D recording (Fig. 3.10). While the ACI map of CaGreenstained ORNs revealed almost identical, but noisier, structures, the ACI map of JG205 stained ORNs clearly showed a morphological distinct compartment.

The number of recovery days after electroporation for animals stained with CaGreen was between 2 and 10 days. Animals that were used for the the initial experiments with JG205 had a comparable recovery time. However, stimulus-induced responses were relatively rare. Only 2 out of 10 animals showed clear responses to stimulation with AA-mix $[100 \mu \mathrm{M}])$. Three additional animals showed weak responses. In contrast, spontaneous events were always observed in JG205-electroporated animals. One spontaneous activity recording was even suitable to separate intraglomerular axons (Fig. 3.11). However, without any stimulus-induced responses it could neither be determined whether this activity proceeded from healthy cells nor whether this particular glomerulus was functional.

In order to separate intraglomerular axons of functional glomeruli located in the lateral cluster, some experimental adjustments had to be made. First of all, I investigated why responses are relatively seldom or weak in the JG205-stained ORNS. To test whether this was related to the different electroporation parameters, I increased the recovery time (more than one week) for JG205-stained animals. Furthermore, to reduce bleaching, laser intensities were reduced. This enabled the recording of multiple stimulus-induced responses, although as a consequence, responses were barely visible during imaging and their amplitudes were reduced. 


\section{Results}
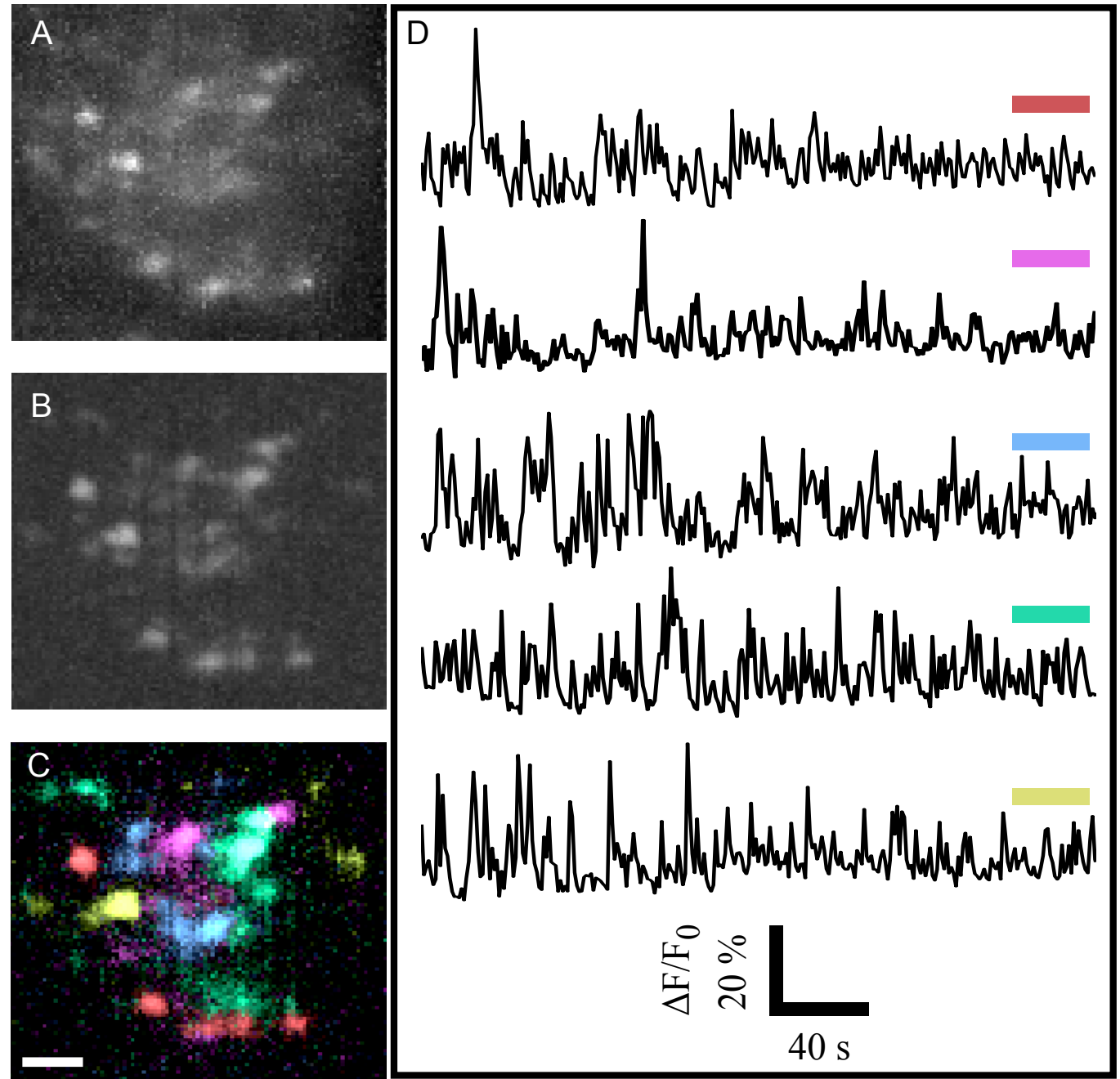

Figure 3.11: Visualization of individual intraglomerular axons.

A: Maximal projection of a JG205-stained glomerulus B: Neighborhood correlation map of A. C: Activity correlation map and in D the reference traces from the components shown here. Scale bar $=10 \mu \mathrm{m}$. 


\section{Results}

After more than one week of recovery time $(n=8)$, responses were observed following stimulation with AA-mix $[100 \mu \mathrm{M}](n=6)$. Responses to AA-mix concentrations of $10 \mu \mathrm{M}$ and lower were observed in those animals as well. In two out of three animals, responses to AA-mix $[100 \mu \mathrm{M}]$ were still observed after increasing the recovery time to more than two weeks.

In parallel to the above described experiments an automatic ROI detection programme was tested on simulated data and compared with ACI. Both in ACI and in the automatic ROI detection, the four distinct activity patterns were detected (Fig. 3.12). ACI used the correlation between reference traces (Fig. 3.12A) and other voxels, whereas the automatic ROI detection program reduced the traces to binary sequences (Fig. 3.12B). In other words, the (overlapping) dots shown in Fig. 3.12B are the event sequences of automatically detected ROIs (y-axis) at frame $i$ (x-axis). Four detected ROIs were comparable to the ROIs detected with ACI (Fig. 3.12C-D).

As a next step, I tested whether the new algorithm could also find multiple ROIs when using experimental data. As mentioned before, stimulus-induced responses were observed in JG205-stained ORNs after a recovery time of more than one week. Those measurements recorded fluctuations of a larger part of the lateral cluster, to increase the success rate of detecting responding glomeruli. I increased the time before the first application, so it was possible to record spontaneous activities as well. Stimulus induced responses were clearly recognizable when comparing the automatically detected sequences. Spontaneous events are detected as well. 


\section{Results}

A

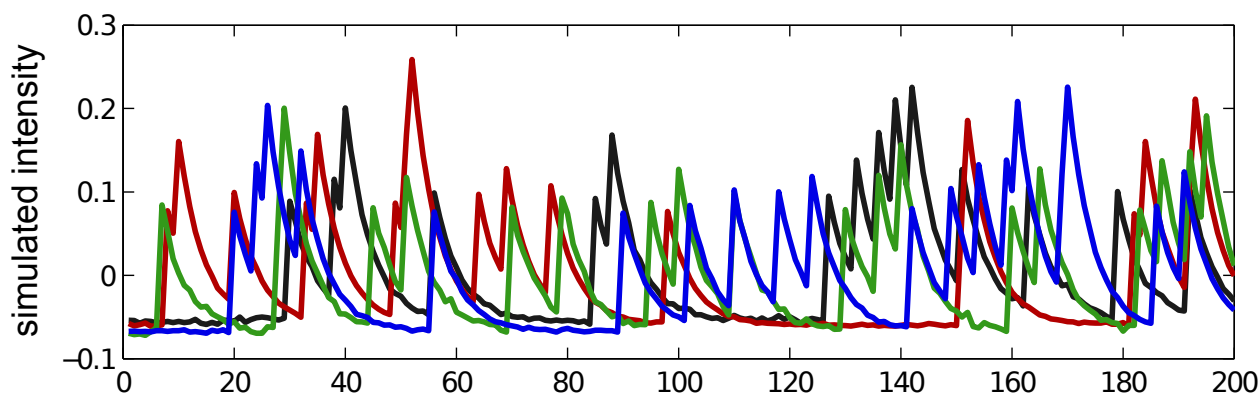

B

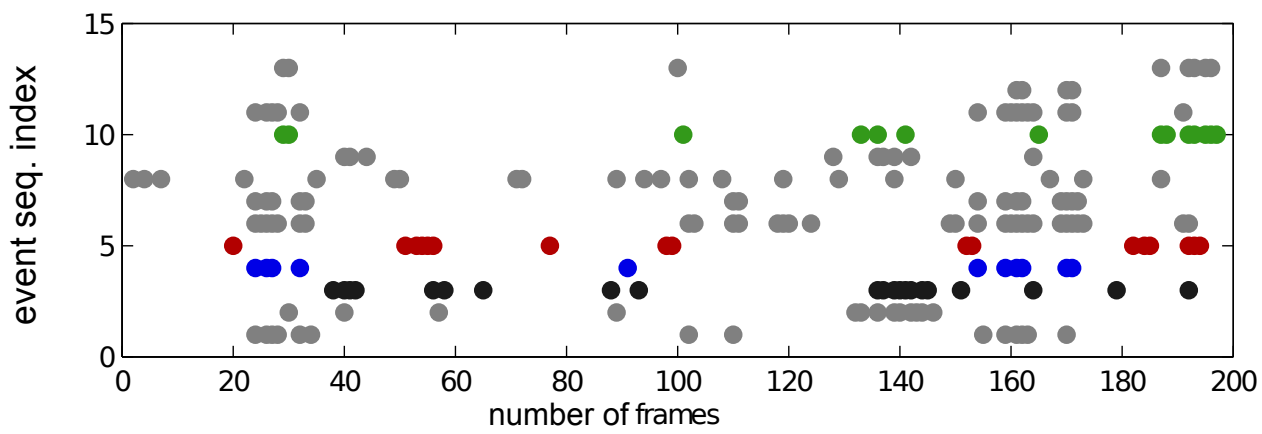

C
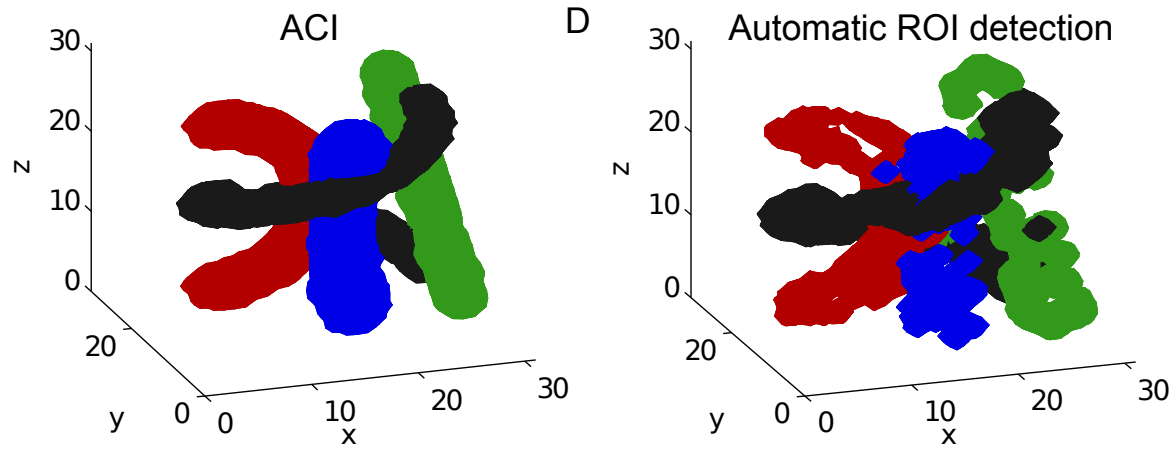

Figure 3.12: Comparison between two ROI selection procedures

A: Four simulated activity traces B: Automatically detected event sequences. C: Semiautomatically detected ROIs. D: Automatically detected ROIs 


\section{Results}

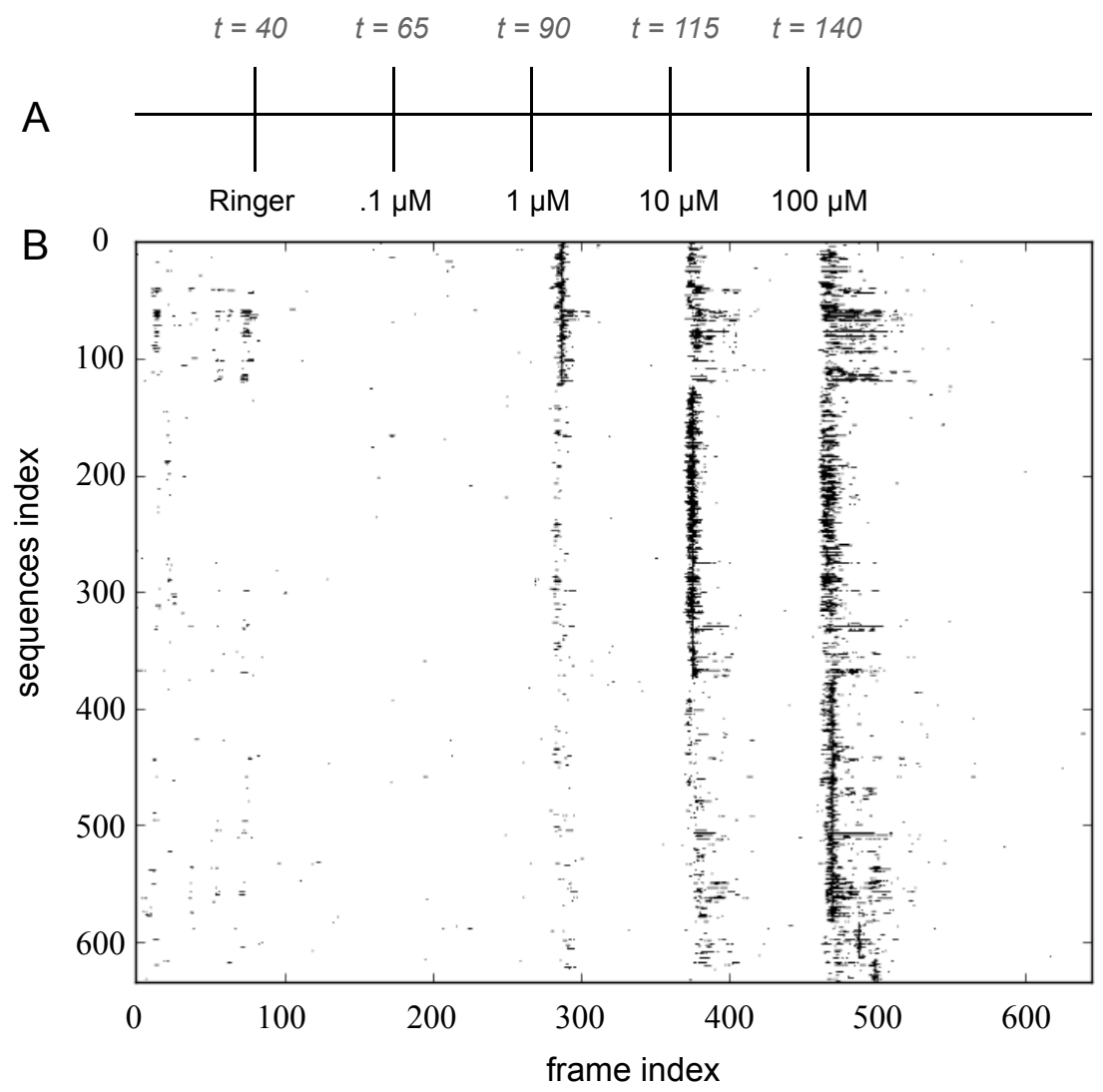

Figure 3.13: Automatic detected sequences from experimental data

A: Stimulus application scheme. After the application of Ringer's solution, AA-mix was applied at different concentrations. Between applications there was an interstimulus interval of 25 seconds. B: listed event sequences. t: time in seconds

It is not easy to detect neuropils using odorant responses alone. However, obtaining morphological information requires an additional recording and would cause bleaching before more relevant information about glomerular activity patterns could be obtained. Yet, morphological data is necessary to reliably select glomeruli. Since the custom built line illuminating microscope had a red laser as well, a second dye was simultaneously electroporated. Thus, morphological data was acquired using the red laser, and at the same time bleaching was prevented. In general, electroporating ORNs with an Alexa 


\section{Results}

dye results in very detailed and bright staining of glomerular axons. However, this was not the case for cells that were simultaneously electroporated with Alexa 680 and JG205. Therefore remained a challenge to select target glomeruli. Ongoing experiments investigate how to define target glomeruli after JG205 electroporation. 


\section{Discussion}

This thesis' work used a combination of recently developed methods to investigate presynaptic activity patterns within individual glomeruli. The results described in chapter 3 show that intraglomerular ORN axons have different sensitivities and spontaneous activity patterns. Characteristic profiles, both the spontaneous and stimulus-induced activities, change over subsequent recordings. Correlated cell-specific activity was required for visualizing individual axons with activity correlation imaging. Once such activity was observed in JG205-stained ORNs. However, the specific glomerulus did not respond to AA-mix stimulation, which may point out that this activity was unphysiological. The low success rate so far, supports this hypothesis. Under physiological conditions, the separation was only partly possible for CaGreen-stained axons.

\subsection{Glomerular sensitivity}

Previously it has been shown that odor response thresholds can be influenced by stimulus application. In flies even the rate of concentration change has been reported to influence response thresholds (Wilson, 2013). This effect is usually explained by stimulusinduced adaptation and/or sensitization. Most of the adaptation studies in olfaction described cAMP-mediated adaptation (Ma and Menini, 2010). However, the majority of amino-acid sensitive cells have a PLC-mediated pathway (Sansone et al., 2014). Our recent data illustrates that the BK-channel plays an important role in shaping odor re- 


\section{Discussion}

sponses (Bao et al., 2015). This channel is known to be involved in olfactory adaptation (Kawai, 2002). Hence, the modulation of the BK channel may play a role in the adaptation of amino-acid sensitive cells. The data presented in this thesis showed that detected response-thresholds changed over time. Control experiments are required to investigate whether this effect is a purely method-dependent effect or whether the variations are related to adaptation processes.

Dose-response experiments revealed that glomeruli can be very sensitive to aminoacids and that both dose-response profiles and spontaneous activity patterns differ over subsequent recordings. Nevertheless, responses were regularly observed after the application of amino acids in the nanomolar range. There are only around $10 \%$ of ORN somata that respond to single amino acids in the nanomalar range (Breunig et al., 2010). Here I used a mixture of those single amino acids. Combined responses, such as summation or hyperaddition may occur (Thomas-Danguin et al., 2014).

Why these effects do not fully explain a sensitivity in the nanomolar range is as follows. First of all we assumed that most of the glomeruli respond to only three or fewer single amino acids (Manzini et al., 2007a). Around $45 \%$ of them respond to only one amino acid (Manzini et al., 2007a). Combining the glomeruli listed in Table 3.1 and Table 3.2, and thereby disregarding any stimulus-induced threshold changes, more than $50 \%$ have a response threshold of $1 \mu \mathrm{M}$ or below. Fifty percent is five times more than the $10 \%$ of ORNs measured at the olfactory epithelium (Breunig et al., 2010). The difference indicates that glomeruli are more sensitive than ORNs. Obviously, it cannot be excluded that if those glomeruli respond to more than a single amino acid, the observed response is the result of an hyperaddition (Thomas-Danguin et al., 2014).

On the other hand, the single amino acid experiments presented in this thesis showed that 3 out of 5 glomeruli were successfully stimulated with $1 \mu \mathrm{M}$ as well (Fig. 3.6). This observation again supports the observation that glomeruli are more sensitive than ORNs. ORNs within glomeruli are probably (almost) mature and only form a subset of the total 


\section{Discussion}

number of ORNs (Manzini et al., 2007a). As a narrowing of odor response profiles is observed over time (Manzini and Schild, 2004), ORNs could become more sensitive to low concentrations as well, which would fit with the current single amino acid data and would explain the somewhat shifted dose response profiles of ORNs measured in the olfactory epithelium. In this line the less sensitive glomerulus shown in Fig. 3.6B may consist of ORNs younger than the more sensitive glomerulus (Fig. 3.6A). An alternative explanation for the difference in sensitivity between the epithelium and the bulb would be that responses are more easily detected in the OB. For example due to differences in $\mathrm{Ca}^{2+}$-channel density and/or a lower diameter of ORN axons, compared to ORN somata. This is supported by the findings in Connelly et al (2013). Their patch clamp recordings revealed stimulus-induced responses in rat ORNs after the application of low-concentrated odors $(10 \mathrm{nM})$. Since rat ORNs are thought to be less sensitive (Duchamp-Viret et al., 2000) than frog ORNs, it could indeed be that the response thresholds of most ORNs are actually lower than those obtained using calcium imaging in the olfactory epithelium.

Dose-response experiments revealed that detecting response thresholds is not a reliable method for the activity-based separation of intraglomerular compartments (Fig. 3.2). On the other hand, the fluctuation of responses observed after the stimulation with lowconcentrated single amino acids confirms the study of Wachowiak et al. (2004) in the sense that response variations throughout glomeruli are observed, especially using low concentrations, and that intraglomerular response profiles cannot be separated using CaGreen. 


\section{Discussion}

\subsection{Odorant responses and spontaneous activity}

During the localization of target glomeruli, spontaneous activity was much more apparent in JG205 stained ORNs than in ORNs electroporated with CaGreen. Although an improved signal-to-noise ratio could contribute to this observation, it may not fully explain the different activity patterns. Since odorant responses were observed more frequently when the recovery time was prolonged, JG205-stained ORNs were probably more damaged after the electroporation protocol. The poor Alexa staining is in line with this hypothesis.

Those results do not exclude that damaged or dying cells have high spontaneous activities. The fact that cells with high spontaneous activity were more apparent in JG205stained ORNs might just depend on the increased signal-to-noise ratio. After CaGreen electroporation there may be a similar number of dying cells, which are just not as easily recognized during imaging. Indeed, also after $\mathrm{CaGreen}$ electroporation several glomeruli of the lateral cluster do not show any responses to amino-acid stimulation (Fig. 3.1).

Based on the results of CaGreen and JG205, I hypothesize that there is an inverted U relationship between age and sensitivity of ORNs. In other words, young cells may be rather insensitive and have a higher response threshold (Fig. 3.6). Cells projecting to glomeruli are probably more mature (Manzini et al., 2007a), which would explain the increased sensitivity of glomeruli (section 3.1). Old or damaged cells will lose this sensitivity again and in those cells a high spontaneous activity was observed as well. The only difference between old and young cells would be that young cells have a broad and old cells a narrow odorant response profile. Future experiments are necessary to test this hypothesis. 


\section{Discussion}

\subsection{Methods of ROI selection}

I compared three different ROI selection procedures to investigate which of them is most suitable for assigning voxels to axons. This section will compare the advantages and disadvantages of those methods regarding the different experimental conditions. ROI selection based on neighborhood correlation maps can only be reliably performed when voxels contain enough correlated events that differ from events of other voxels. Since stimulus-induced responses between different intraglomerular regions at least partly overlap in time, ACI maps of different ROIs will always reveal all regions that reacted to this stimulus. Regarding spontaneous activity, those events are too few and have a low signal-to-noise ratio, which results in a very noisy neighborhood correlation map. Neither stimulus-induced responses nor spontaneous activity traces of CaGreenstained ORNs are suitable for a purely correlation dependent analysis. Using manual ROI selection, differences between spontaneous activity patterns and differences in response profiles were observed. Yet, the fact that structures appear or disappear over recordings, due to bleaching or z-movement, constrains manual ROI selection, which is one of the disadvantages of this method. Furthermore, ROIs drawn manually, probably covered axons that were very close to each other.

The limitations of manual and semi-automatic ROI detection motivated us to develop an automatic ROI selection programme for the analysis of activity patterns of CaGreenstained glomeruli. Here, the first applications of this algorithm show that regions revealed wit ACI are also detected as individual ROIs after running the new programme. An automatic ROI detection procedure has several advantages compared to ACI. First of all, the automatic ROI selection is less prone to experimental bias, since no manual steps are required. Secondly, the experimenter can define system-specific criteria to distinguish traces. For example, whether sequences should contain at least $n$ nonoverlapping events before they are assigned to a different ROI (see section 2.6.3 for other parameters). This flexibility is a huge advantage in comparison to ACI and the 


\section{Discussion}

algorithm is suitable for distinguishing intraglomerular axons using a voxel-based analysis of activity patterns. On the other hand, this flexibility can easily lead to false positive or false negative ROIs. Especially when the knowledge about event fluctuations within single cells is limited. This will complicate the choice of suitable parameters. The presented results showed that the kind of activity will define the appropriate ROIselection procedure. When the number of events across multiple axons is low and some of them are even correlated, they can probably not be distinguished with ACI. By contrast, when there are many events or the event intervals are short ACI will probably be the most suitable approach. At this moment it is not known to what extent uncorrelated events can occur in the axon terminals of one ORN. One physiological explanation for such events would be the spontaneous opening of $\mathrm{Ca}^{2+}$ channels. Future experiments, for example calcium imaging in axon terminals of single-stained ORNs, are required to investigate uncorrelated events in single cells and to what extent they possibly bias the activity-based separation of individual intraglomerular axons. 


\section{Conclusions and future directions}

The three main points of this thesis are the following. Glomeruli can be very sensitive and fluctuations of stimulus-induced responses constrain axon separation based on stimulus-induced response profiles (1). By comparing spontaneous activity, but not stimulus-induced responses, coarse intraglomerular compartments were identified using CaGreen (2). Individual ORN axons were revealed by activity correlation imaging only whenever enough correlated cell-specific activity was obtained (3), for example by using JG205. The combination of fast 3D calcium imaging and ACI enables the study of presynaptic odor representation within and between individual axons of single glomeruli. This combination of techniques is also applicable in other research areas focusing on signal processing in dense networks and the contributions of single neurons. 


\section{Summary}

Olfactory glomeruli are structural and functional neuropils in the olfactory bulb. Although many olfactory receptor neurons (ORNs) project to one glomerulus, stimulusinduced responses within single glomeruli could not be previously distinguished using calcium imaging. At the level of the olfactory epithelium, however, activity differences within ORN subpopulations have been reported.

I studied stimulus-induced and spontaneous activity patterns of intraglomerular ORN axons in order to obtain a deeper understanding of presynaptic odor representations within single glomeruli. Dose-response profiles and spontaneous activity patterns were obtained for glomeruli stained with CaGreen or JG205, which is a newly synthesized dye. From CaGreen-stained glomeruli odorant response profiles were acquired as well. Activity-specific information was then used to investigate how individual intraglomerular axons can be distinguished based on activity patterns.

The presented data revealed, that, a combination of fast 3D line-illumination microscopy and activity correlation of spontaneous activity patterns was sufficient for the visualization of JG205-stained ORN axons. An activity-based separation of intraglomerular axons was, however, only partially possible for axons stained with CaGreen. The latter confirms previously reported results, namely, that response profiles and/or spontaneous activities from CaGreen-stained glomeruli cannot be assigned to individual CaGreen-stained axons (Wachowiak et al., 2004). 


\section{Summary}

The study of information processing within individual intraglomerular axons is essential for a deeper understanding of the presynaptic representation of odors. Visualizing and identifying individual intraglomerular axons simultaneously based on their activity patterns facilitates the study of presynaptic information processing. Furthermore, the combination of fast 3D calcium imaging and activity correlation imaging can also be used to investigate information processing in single neurons across brain areas and species. 


\section{References}

Aimé, P., P. Duchamp-Viret, M. A. Chaput, A. Savigner, M. Mahfouz, and A. K. Julliard (2007). "Fasting increases and satiation decreases olfactory detection for a neutral odor in rats." Behav Brain Res 179.2, pp. 258-264.

Alevra, M. (2013). "Basic coding activities of populations of Xenopus laevis olfactory receptor neurons recorded with a fast confocal line illumination microscope". PhD thesis.

Antunes, G., A. M. Sebastião, and F. M. Simoes de Souza (2014). "Mechanisms of regulation of olfactory transduction and adaptation in the olfactory cilium." PLoS One 9.8, e105531.

Bao, G., D. de Jong, M. Alevra, and D. Schild (2015). "Ca(2+) /BK channel clusters in olfactory receptor neurons and their role in odor coding." Eur J Neurosci.

Bao, G. and D. Schild (2014). "Fast and accurate fitting and filtering of noisy exponentials in Legendre space." PLoS One 9.3, e90500.

Bozza, T., P. Feinstein, C. Zheng, and P. Mombaerts (2002). "Odorant receptor expression defines functional units in the mouse olfactory system." J Neurosci 22.8, pp. 3033-3043.

Braubach, O. R., A. Fine, and R. P. Croll (2012). "Distribution and functional organization of glomeruli in the olfactory bulbs of zebrafish (Danio rerio)." J Comp Neurol 520.11, 2317-39, Spc1. 
Breunig, E., I. Manzini, F. Piscitelli, B. Gutermann, V. Di Marzo, D. Schild, and D. Czesnik (2010). "The endocannabinoid 2-arachidonoyl-glycerol controls odor sensitivity in larvae of Xenopus laevis.” J Neurosci 30.26, pp. 8965-8973.

Buck, L. and R. Axel (1991). “A novel multigene family may encode odorant receptors: a molecular basis for odor recognition." Cell 65.1, pp. 175-187.

Byrd, C. A. and G. D. Burd (1991). "Development of the Olfactory Bulb in the Clawed Frog, Xenopus laevis: A Morphological and Quantative Analysis”. The Journal of Comparative Neurology 314, pp. 79-90.

Cadiou, H., I. Aoudé, B. Tazir, A. Molinas, C. Fenech, N. Meunier, and X. Grosmaitre (2014). "Postnatal odorant exposure induces peripheral olfactory plasticity at the cellular level." J Neurosci 34.14, pp. 4857-4870.

Chen, T.-W., B.-J. Lin, and D. Schild (2009). "Odor coding by modules of coherent mitral/tufted cells in the vertebrate olfactory bulb." Proc Natl Acad Sci U S A 106.7, pp. 2401-2406.

Cleland, T. A., B. A. Johnson, M. Leon, and C. Linster (2007). "Relational representation in the olfactory system." Proc Natl Acad Sci U S A 104.6, pp. 1953-1958.

Connelly, T., A. Savigner, and M. Ma (2013). "Spontaneous and sensory-evoked activity in mouse olfactory sensory neurons with defined odorant receptors." J Neurophysiol 110.1, pp. 55-62.

Couto, A., M. Alenius, and B. J. Dickson (2005). "Molecular, anatomical, and functional organization of the Drosophila olfactory system." Curr Biol 15.17, pp. 1535-1547.

Duchamp-Viret, P., A. Duchamp, and M. A. Chaput (2000). "Peripheral odor coding in the rat and frog: quality and intensity specification." J Neurosci 20.6, pp. 2383-2390.

Elsaesser, R. and J. Paysan (2007). “The sense of smell, its signalling pathways, and the dichotomy of cilia and microvilli in olfactory sensory cells." BMC Neurosci 8 Suppl $3, \mathrm{~S} 1$. 
Feinstein, P., T. Bozza, I. Rodriguez, A. Vassalli, and P. Mombaerts (2004). “Axon guidance of mouse olfactory sensory neurons by odorant receptors and the beta 2 adrenergic receptor." Cell 117.6, pp. 833-846.

Gliem, S., A. S. Syed, A. Sansone, E. Kludt, E. Tantalaki, T. Hassenklöver, S. I. Korsching, and I. Manzini (2013). "Bimodal processing of olfactory information in an amphibian nose: odor responses segregate into a medial and a lateral stream." Cell Mol Life Sci 70.11, pp. 1965-1984.

Grosmaitre, X., A. Vassalli, P. Mombaerts, G. M. Shepherd, and M. Ma (2006). "Odorant responses of olfactory sensory neurons expressing the odorant receptor MOR23: a patch clamp analysis in gene-targeted mice." Proc Natl Acad Sci U S A 103.6, pp. 1970-1975.

Hassenklöver, T. and I. Manzini (2013). "Olfactory wiring logic in amphibians challenges the basic assumptions of the unbranched axon concept." J Neurosci 33.44, pp. 17247-17252.

- (2014). "The olfactory system as a model to study axonal growth patterns and morphology in vivo." J Vis Exp 92, e52143.

Hildebrand, J. G. and G. M. Shepherd (1997). "Mechanisms of olfactory discrimination: converging evidence for common principles across phyla." Annu Rev Neurosci 20, pp. 595-631.

Imai, T., H. Sakano, and L. B. Vosshall (2010). "Topographic mapping-the olfactory system.” Cold Spring Harb Perspect Biol 2.8, a001776.

Imai, T., M. Suzuki, and H. Sakano (2006). “Odorant receptor-derived cAMP signals direct axonal targeting." Science 314.5799, pp. 657-661.

Junek, S., T.-W. Chen, M. Alevra, and D. Schild (2009). "Activity correlation imaging: visualizing function and structure of neuronal populations." Biophys $J$ 96.9, pp. 3801-3809. 
Junek, S., E. Kludt, F. Wolf, and D. Schild (2010). “Olfactory coding with patterns of response latencies." Neuron 67.5, pp. 872-884.

Kawai, F. (2002). "Ca2+-activated K+ currents regulate odor adaptation by modulating spike encoding of olfactory receptor cells.” Biophys $J$ 82.4, pp. 2005-2015.

Kishida, T., J. Thewissen, S. Usip, R. S. Suydam, and J. C. George (2015). "Organization and distribution of glomeruli in the bowhead whale olfactory bulb." PeerJ 3, e897.

Kurahashi, T. and A. Menini (1997). "Mechanism of odorant adaptation in the olfactory receptor cell." Nature 385.6618, pp. 725-729.

Ma, M. and A. Menini (2010). "Multiple olfactory subsystems convey various sensory signals". The neurobiology of olfaction, pp. 225-240.

Manzini, I., C. Brase, T.-W. Chen, and D. Schild (2007a). "Response profiles to amino acid odorants of olfactory glomeruli in larval Xenopus laevis." J Physiol 581.Pt 2, pp. 567-579.

Manzini, I., S. Heermann, D. Czesnik, C. Brase, D. Schild, and W. Rössler (2007b). "Presynaptic protein distribution and odour mapping in glomeruli of the olfactory bulb of Xenopus laevis tadpoles.” Eur J Neurosci 26.4, pp. 925-934.

Manzini, I., F. Peters, and D. Schild (2002a). "Odorant responses of Xenopus laevis tadpole olfactory neurons: a comparison between preparations." J Neurosci Methods 121.2, pp. 159-167.

Manzini, I., W. Rössler, and D. Schild (2002b). "cAMP-independent responses of olfactory neurons in Xenopus laevis tadpoles and their projection onto olfactory bulb neurons." J Physiol 545.Pt 2, pp. 475-484.

Manzini, I. and D. Schild (2004). "Classes and narrowing selectivity of olfactory receptor neurons of Xenopus laevis tadpoles.” J Gen Physiol 123.2, pp. 99-107. 
Martelli, C., J. R. Carlson, and T. Emonet (2013). "Intensity invariant dynamics and odor-specific latencies in olfactory receptor neuron response.” J Neurosci 33.15, pp. 62856297.

Meister, M. and T. Bonhoeffer (2001). "Tuning and topography in an odor map on the rat olfactory bulb." J Neurosci 21.4, pp. 1351-1360.

Mombaerts, P. (2006). “Axonal wiring in the mouse olfactory system.” Annu Rev Cell Dev Biol 22, pp. 713-737.

Moriya-Ito, K., I. Tanaka, Y. Umitsu, M. Ichikawa, and H. Tokuno (2015). “The olfactory bulb and the number of its glomeruli in the common marmoset (Callithrix jacchus)." Neurosci Res 93, pp. 158-163.

Murmu, M. S., J. Stinnakre, E. Réal, and J.-R. Martin (2011). "Calcium-stores mediate adaptation in axon terminals of olfactory receptor neurons in Drosophila." $B M C$ Neurosci 12, p. 105.

Nezlin, L. P., S. Heermann, D. Schild, and W. Rössler (2003). “Organization of glomeruli in the main olfactory bulb of Xenopus laevis tadpoles." J Comp Neurol 464.3, pp. 257 268.

Nezlin, L. P. and D. Schild (2005). "Individual olfactory sensory neurons project into more than one glomerulus in Xenopus laevis tadpole olfactory bulb." J Comp Neurol 481.3, pp. 233-239.

Nieuwkoop, P. and J. Faber (1994). NORMAL TABLE OF XENOUPUS LAEVIS (DAUDIN). Ed. by P. Nieuwkoop and J. Faber. GARLAND PUBLISHING, INC. New York \& London.

Pifferi, S., A. Menini, and T. Kurahashi (2009). "Signal transduction in vertebrate olfactory cilia”. The Neurobiology of Olfaction 28, p. 203.

Rawal, S., H. J. Hoffman, A. K. Chapo, and V. B. Duffy (2014). "Sensitivity and Specificity of Self-Reported Olfactory Function in a Home-Based Study of IndependentLiving, Healthy Older Women." Chemosens Percept 7.3-4, pp. 108-116. 
Reisert, J. and H. Zhao (2011). "Perspectives on: information and coding in mammalian sensory physiology: response kinetics of olfactory receptor neurons and the implications in olfactory coding." J Gen Physiol 138.3, pp. 303-310.

Rospars, Lánský, Duchamp-Viret, and Duchamp (2000). "Spiking frequency versus odorant concentration in olfactory receptor neurons." Biosystems 58.1-3, pp. 133141.

Rospars, Sanda, Lansky, and Duchamp-Viret (2013). "Responses of single neurons and neuronal ensembles in frog first- and second-order olfactory neurons." Brain Res 1536, pp. 144-158.

Sansone, A., T. Hassenklöver, A. S. Syed, S. I. Korsching, and I. Manzini (2014). "Phospholipase $\mathrm{C}$ and diacylglycerol mediate olfactory responses to amino acids in the main olfactory epithelium of an amphibian.” PLoS One 9.1, e87721.

Savigner, A., P. Duchamp-Viret, X. Grosmaitre, M. Chaput, S. Garcia, M. Ma, and B. Palouzier-Paulignan (2009). "Modulation of spontaneous and odorant-evoked activity of rat olfactory sensory neurons by two anorectic peptides, insulin and leptin." $J$ Neurophysiol 101.6, pp. 2898-2906.

Schild, D. and D. Restrepo (1998). "Transduction mechanisms in vertebrate olfactory receptor cells." Physiol Rev 78.2, pp. 429-466.

Song, Y., K. D. Cygnar, B. Sagdullaev, M. Valley, S. Hirsh, A. Stephan, J. Reisert, and H. Zhao (2008). "Olfactory CNG channel desensitization by $\mathrm{Ca} 2+/ \mathrm{CaM}$ via the B1b subunit affects response termination but not sensitivity to recurring stimulation." Neuron 58.3, pp. 374-386.

Soucy, E. R., D. F. Albeanu, A. L. Fantana, V. N. Murthy, and M. Meister (2009). "Precision and diversity in an odor map on the olfactory bulb." Nat Neurosci 12.2, pp. 210-220. 
Spehr, J., S. Hagendorf, J. Weiss, M. Spehr, T. Leinders-Zufall, and F. Zufall (2009). " $\mathrm{Ca} 2+$-calmodulin feedback mediates sensory adaptation and inhibits pheromonesensitive ion channels in the vomeronasal organ." J Neurosci 29.7, pp. 2125-2135.

Stephan, A. B., S. Tobochnik, M. Dibattista, C. M. Wall, J. Reisert, and H. Zhao (2012). "The $\mathrm{Na}(+) / \mathrm{Ca}(2+)$ exchanger NCKX4 governs termination and adaptation of the mammalian olfactory response." Nat Neurosci 15.1, pp. 131-137.

Takeuchi, H. and H. Sakano (2014). "Neural map formation in the mouse olfactory system.” Cell Mol Life Sci 71.16, pp. 3049-3057.

Thomas-Danguin, T., C. Sinding, S. Romagny, F. El Mountassir, B. Atanasova, E. Le Berre, A.-M. Le Bon, and G. Coureaud (2014). "The perception of odor objects in everyday life: a review on the processing of odor mixtures." Front Psychol 5, p. 504.

Valle-Leija, P. (2015). “Odorant Receptors Signaling Instructs the Development and Plasticity of the Glomerular Map.” Neural Plast 2015, p. 975367.

Wachowiak, M. and L. B. Cohen (2001). "Representation of odorants by receptor neuron input to the mouse olfactory bulb." Neuron 32.4, pp. 723-735.

Wachowiak, M., W. Denk, and R. W. Friedrich (2004). "Functional organization of sensory input to the olfactory bulb glomerulus analyzed by two-photon calcium imaging." Proc Natl Acad Sci U S A 101.24, pp. 9097-9102.

Wachowiak, M., J. P. McGann, P. M. Heyward, Z. Shao, A. C. Puche, and M. T. Shipley (2005). "Inhibition [corrected] of olfactory receptor neuron input to olfactory bulb glomeruli mediated by suppression of presynaptic calcium influx." J Neurophysiol 94.4, pp. 2700-2712.

Wilson, R. I. (2013). "Early olfactory processing in Drosophila: mechanisms and principles." Annu Rev Neurosci 36, pp. 217-241.

Yu, Y., N. P. Boyer, and C. Zhang (2014). "Three structurally similar odorants trigger distinct signaling pathways in a mouse olfactory neuron." Neuroscience 275, pp. 194 210. 
Zou, D.-J., P. Feinstein, A. L. Rivers, G. A. Mathews, A. Kim, C. A. Greer, P. Mombaerts, and S. Firestein (2004). "Postnatal refinement of peripheral olfactory projections." Science 304.5679, pp. 1976-1979. 


\section{Acknowledgements}

First of all, I am grateful for the opportunity to work as a PhD student in the lab of Prof. Dr. Dr. Detlev Schild. Professor Schild, I am really thankful for your confidence in me and my work. Thank you for sharing your experience, your insights and extraordinary knowledge. It helped me to become a better and independent researcher.

I would like to thank all my colleagues and students who work(ed) in the lab. Thank you for your help, criticism, your strong opinions, but above all thank you for providing a collegial environment. Alexander, Angie, Camille, Bernd and Mihai, thank you for your advise and your corrections of the different parts of my thesis.

I am grateful to the various teachers and mentors, who guided me during the last few years. I would like to thank my thesis committee members, Prof. Dr. Michael Müller and Prof. Dr. Walter Stühmer. Thank you for your critical questions, your proposals and your time. Also thanks to Prof. Dr. Martin Göpfert, Prof. Dr. Ernst Wimmer and Prof. Dr. André Fiala for joining my examination board.

Dr. Heike Conrad and Prof. Dr. Matthias Dobbelstein, I appreciate your availability and reliability whenever I needed it. Thank you very much for your support. You were always able to put the things that bothered me in the right perspective.

I would like to thank the GGNB management team and the GGNB board. Thanks to you I had the opportunity to be part a very stimulating and exciting research environment, not only as a $\mathrm{PhD}$ student, but also as the CMPB student representative. Prof. Dr. Michael Hörner, thank you for organizing the CMPB seminars. The critical 


\section{Acknowledgements}

discussions and our personal communication contributed to my progress over the last years.

Last but not least I would like to thank my friends, my family and my partner. Without my friends I would not have come so far. Special thanks go to Angie, Lilith, Julia, Hans and Thorsten. You are all very different, but having you around is just amazing.

I am thankful to the members of KlezPO and the Kitchen Band. I would like to especially thank Wieland, Claudia, and Howard. Wieland, your motivation-strategies are great. I am still running. Claudia, you are a real example for me. Your flexibility, reliability, your ambitions, your insights and your love for music are just wonderful. Howard, I loved the hours playing Klezmer together with the Kitchen Band in your kitchen. Of course I would also like to thank you for making time to proof read my thesis.

Ik wil mijn familie bedanken voor al de ondersteunende gesprekken, de bemoedigende kaartjes, de "zomaar" mailtjes en natuurlijk voor hun gebeden. Daniel, fijn dat je er altijd voor me bent. Je zorgzaamheid heeft er toe geleid dat ik keer op keer nieuwe kracht vond om door te gaan. 


\section{Curriculum Vitae}

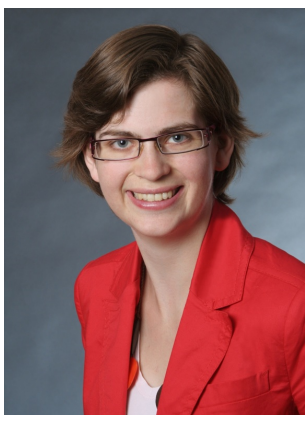
NAME:
DANIËLLE DE JONG
Address: $\quad$ Bergstraße 8
City: $\quad 31167$ BOCKENEM
Phone: $\quad+49(0) 1767092537$
Date of Birth: November 1, 1987
Place of Birth: Dordrecht
Citizenship: Dutch
Sex: $\quad$ Female

\section{EDUCATION}

$\begin{array}{rll}\text { 12/2011 - Present: } & \text { GGNB Student } \\ & \text { Göttingen Graduate School for Neurosciences } \\ & \text { Biophysics and Molecular Biosciences } \\ 09 / 2011-\text { Present: } & \text { PhD Candidate } \\ & \text { Institute of Neurophysiology and } \\ & \text { Cellular Biophysics } \\ 12 / 2012-07 / 2014 & \text { Mentoring - Programm } \\ & \text { Universitätsmedizin Göttingen } \\ 09 / 2008-11 / 2010 & \text { Master Brain and Cognitive Sciences } \\ & \text { Cognitive Science Centre of Amsterdam } \\ 09 / 2005-10 / 2008 & \text { Bachelor Psychobiology } \\ & \text { University of Amsterdam }\end{array}$

EMPLOYMENT HISTORY

Administrative Assistent

\begin{tabular}{|c|c|c|}
\hline $11 / 2010$ & - $07 / 2011$ & IHC Dredgers B.V. \\
\hline \multirow[t]{2}{*}{$11 / 2007$} & - $03 / 2008$ & Suitcase talen \\
\hline & & Almere \\
\hline \multirow[t]{2}{*}{$05 / 2006$} & $-12 / 2007$ & Blanken Medisch \\
\hline & & Adviseurs \\
\hline \multirow[t]{2}{*}{$05 / 2005$} & - 07/2005 & Primary School \\
\hline & & De Loopplank \\
\hline \multicolumn{3}{|c|}{ TEACHING } \\
\hline \multicolumn{3}{|c|}{ Practical Course Neurophysiology } \\
\hline
\end{tabular}

\section{RESEARCH PROJECTS}

09/2011 - 06/2015 Institute of Neurophysiology and Cellular Biophysics

Project: Glomerular Information Processing in Xenopus laevis

11/2009 - 08/2010 Swammerdam Institute for Life Sciences Department Cognitive Neurosciences

Project 1 Dendritic morphology of hippocampal neurons in mice

Project 2 Acitivity dependent effects of stresshormones on AMPA receptor immunolabeling

01/2009 - 07/2009 Netherlands Institute for Neuroscience

Project: Thyroid hormone effects on hyptohalamic signaling 


\section{Curriculum Vitae}

\section{MASTER LITERATURE THESIS}

Norepinephrine and Emotional Memory

\section{PUBLICATIONS}

Pillai, AG., de Jong, D., Kanatsou, S., Krugers, H., Knapman, A., Heinzmann, JM., et al. (2012).

Dendritic morphology of hippocampal and amygdalar neurons in adolescent mice is resiliant to genetic differences in stress reactivity. PloS One 7(6): e38971

Bao, G., de Jong, D., Alevra, M., Schild, D. (2015).

$\mathrm{Ca}^{2+}$-BK channel clusters in olfactory receptor neurons and their role in odour coding.

European Journal of Neuroscience 42(11): 2985-95

SKILLS:

Software: $\quad$ Latex, Matlab, Python, Inkscape, Microsoft Office

Languages: $\quad$ English, German, Dutch (Mothertongue)

Methods: $\quad$ Calcium Imaging, Immunohistochemistry, Fluorescence Microscopy, Patch-clamp

\section{HOBBIES AND EXTRACURRICULAR ACTIVITIES}

02/2016 - Present: Board of the Klezmer Project Orchestra

1997 - Present: Playing soprano saxophone in different orchestras and bands

03/2012 - 10/2013: Student Representative CMPB program of GGNB

Organization of yearly student retreat and biennial symposium

01/2010 - 12/2010: Chairwoman organizing a musical fun fare with workshops, catering and concerts 